Preprint typeset in JHEP style. - HYPER VERSION

J. High Energy Phys. 12 (1999) 010

hep-th/9911093

\title{
Dyons and Interactions in Nonlinear (Born-Infeld) Electrodynamics
}

\author{
by Alexander A. Chernitskii \\ St.Petersburg Electrotechnical University, \\ Prof. Popov str. 5, St.Petersburg 197376, Russia \\ E-mail: aa@cher.etu.spb.ru
}

\begin{abstract}
Born-Infeld nonlinear electrodynamics with point singularities having both electric and magnetic charges are considered. Problem of interaction between the associated soliton dyon solutions is investigated. For the case of long-range interaction at first order by a small field of distant solitons we obtain that the generalized Lorentz force is acted on a dyon under consideration. Short-range interaction between two dyons having identical electric and opposite magnetic charges is investigated for an initial approximation. We consider the case when the velocities of the dyons have equal modules and opposite directions on a common line. It is shown that the associated field configuration has a constant full angular momentum which is independent of the interdyonic distance and their speed. This property permits a consideration of this bidyon configuration as an electromagnetic model of charged particle with spin. We numerically investigate movement of the dyons in this configuration for the case when the full electric charge equals the electron charge and the full angular momentum equals the electron spin. It is shown that for this case the absolute value of relation between electric and magnetic charges of the dyons equals the fine structure constant. The calculation gives that the bidyon may behave as nonlinear oscillator. Associated dependence of frequency on the full energy is obtained for the initial approximation. In the limits of the electrodynamic model we obtain that the quick-oscillating wave packet may behave like massive gravitating particle when it move in high background field. We discuss the possible electrodynamic world with the oscillating bidyons as particles.
\end{abstract}




\section{Contents}

1. Introduction 1

2. Basic relation for field 2

3. Boundary conditions at the singular points 5

4. Dyon solutions and singularities 6

5. Variational principle for two potentials 7

6. Conservation laws and comments about dimensions 10

7. Method for investigation of interaction between dyons 11

8. Interaction of dyon with a small given field 14

9. Bidyon or an electromagnetic model of particle with spin 16

10. Effect of gravitational interaction 28

11. Nonlinear electrodynamic world with oscillating bidyons 32

12. Conclusions 33

\section{Introduction}

Solitons in nonlinear electrodynamics may behave like real particles. This analogy with classical particles is manifested when we consider a long-range interaction of solitons by a perturbation method [1, 2]. In this case a soliton is subjected to the Lorentz force in the first order by a field of distant solitons. The second order may give the soliton's trajectory in the form of geodesic line for some effective Riemann space with metric depending on the field of distant solitons. Thus we have also an analogy with gravitating particles. Moreover, light beams distortion under the action of some given field may appear as gravitational distortion [3]. These properties (in particular) provoke interest to nonlinear electrodynamic models in the context of possible unifying description for the real material world. In the framework of 
this approach it may hope to solve the problem of unification electromagnetism and gravitation.

Here we shall consider an interaction of singular solitons in Born-Infeld nonlinear electrodynamics ${ }^{1}$. The investigation of this nonlinear electrodynamic model with point singularities of field is presented in the article [6], where we have considered the singularities with electric charge. But the model's system of equations for electromagnetic field has an exact solution which has non-zero divergence for both fields of electric D and magnetic B inductions at the singular point. That is, this point for the solution has both electric and magnetic charges. The particle with both electric and magnetic charges had been named by J. Schwinger as dyon (see [7]). Because the singular solution looks like point charged particle [6], we name this field configuration as dyon. In the present article we consider an interaction between these dyons and we consider some quick-oscillating two-dyons field configuration. We also consider like-gravitational interaction of a quick-oscillating wave packet with given field.

\section{Basic relation for field}

Let us state the basic relation for the Born-Infeld nonlinear electrodynamics with singularities [6] generalized to the case with both electric and magnetic charges. We shall use an inertial coordinate system in Minkowskian space: $\left\{x^{\mu}\right\}$ (the Greek indexes take values $0,1,2,3)$. That is, components of metric is independent of time $x^{0}$ and $\left|g_{00}\right|=1, g_{0 i}=0$ (the Latin indexes take values $1,2,3$ ). In this case (see also [6]) we can write the following nonlinear Maxwell system of equations:

$$
\left\{\begin{array}{r}
\operatorname{div} \mathbf{B}=4 \pi \bar{\jmath}^{0} \\
\operatorname{div} \mathbf{D}=4 \pi \jmath^{0}
\end{array}\right\},
$$

where

$$
\begin{aligned}
& \left\{\begin{array}{l}
\mathbf{D}=\frac{1}{\mathcal{L}}\left(\mathbf{E}+\alpha^{2} \mathcal{J} \mathbf{B}\right) \\
\mathbf{H}=\frac{1}{\mathcal{L}}\left(\mathbf{B}-\alpha^{2} \mathcal{J} \mathbf{E}\right)
\end{array}, \quad\left\{\begin{array}{l}
\mathbf{E}=\frac{1}{\overline{\mathcal{L}}}\left(\mathbf{D}-\alpha^{2} \mathcal{J} \mathbf{H}\right) \\
\mathbf{B}=\frac{1}{\overline{\mathcal{L}}}\left(\mathbf{H}+\alpha^{2} \mathcal{J} \mathbf{D}\right)
\end{array}\right.\right. \\
& \mathcal{L}=\sqrt{\left|1-\alpha^{2} \mathcal{I}-\alpha^{4} \mathcal{J}^{2}\right|}, \quad \mathcal{I}=\mathbf{E}^{2}-\mathbf{B}^{2} \quad, \quad \mathcal{J}=\mathbf{E} \cdot \mathbf{B}, \\
& \overline{\mathcal{L}} \equiv \sqrt{\left|1-\alpha^{2} \overline{\mathcal{I}}-\alpha^{4} \overline{\mathcal{J}}^{2}\right|} \quad, \quad \overline{\mathcal{I}}=\mathbf{H}^{2}-\mathbf{D}^{2} \quad, \quad \overline{\mathcal{J}}=\mathbf{H} \cdot \mathbf{D} \text {, }
\end{aligned}
$$

\footnotetext{
${ }^{1}$ It will be recalled that M. Born and L. Infeld had been considered in their article 1 the electrodynamic model which follows from an action that had been proposed by A.S. Eddington in his book 河.
} 
$\jmath^{\mu}$ and $\bar{\jmath}^{\mu}$ are components of electric and magnetic singular currents such that

$$
\begin{array}{rlrl}
\jmath^{0} & \equiv \frac{1}{\sqrt{|g|}} \sum_{n=1}^{N} \stackrel{n}{d} \delta(\mathbf{x}-\stackrel{n}{\mathbf{a}}) & , & \boldsymbol{\jmath} \equiv \frac{1}{\sqrt{|g|}} \sum_{n=1}^{N} \stackrel{n}{d} \stackrel{n}{\mathbf{V}} \delta(\mathbf{x}-\stackrel{n}{\mathbf{a}}) \\
\bar{\jmath}^{0} \equiv \frac{1}{\sqrt{|g|}} \sum_{n=1}^{N} \stackrel{n}{b} \delta(\mathbf{x}-\mathbf{a}) & , & \overline{\boldsymbol{\jmath}} \equiv \frac{1}{\sqrt{|g|}} \sum_{n=1}^{N} \stackrel{n}{b} \stackrel{n}{\mathbf{V}} \delta(\mathbf{x}-\stackrel{n}{\mathbf{a}})
\end{array}
$$

$\stackrel{n}{d}$ and $\stackrel{n}{b}$ are electric and magnetic charges of $n$-th singularity,

$\stackrel{n}{\mathbf{a}}=\stackrel{n}{\mathbf{a}}\left(x^{0}\right)$ is a trajectory of it and $\stackrel{n}{\mathbf{V}} \equiv \frac{\mathrm{d} \mathbf{a}}{\mathrm{d} x^{0}}$.

Here we use the definition for the three-dimensional $\delta$-function which is suitable for discontinuous functions $f(\mathbf{x})$ :

$$
\int_{\substack{n \\ \Omega}} f(\mathbf{x}) \delta(\mathbf{x}-\mathbf{a})(\mathrm{d} x)^{3} \equiv \lim _{\substack{n \\ \sigma \rightarrow 0}}\left\{\frac{1}{||^{n} \mid} \int_{\substack{n \\ \sigma}} f(\mathbf{x}) \mathrm{d} \stackrel{n}{\sigma}\right\} \equiv\langle f(\mathbf{x})\rangle_{n},\left|\sigma^{n}\right| \equiv \int_{\substack{n \\ \sigma}} \mathrm{d} \sigma^{n}
$$

where $\stackrel{n}{\Omega}$ is a region of three-dimensional space including the point $\mathbf{x}=\stackrel{n}{\mathbf{a}}$, $\stackrel{n}{\sigma}$ is a closed surface enclosing this point, $\mathrm{d} \stackrel{n}{\sigma}$ is an area element of the surface $\stackrel{n}{\sigma}$, $|\stackrel{n}{\sigma}|$ is an area of the whole surface $\stackrel{n}{\sigma}$.

Let us define the following functions:

$$
\begin{aligned}
\mathcal{H} & \equiv \mathcal{L}+\alpha^{2} \mathbf{D} \cdot \mathbf{E}=\overline{\mathcal{L}}+\alpha^{2} \mathbf{B} \cdot \mathbf{H}=\alpha^{2} T^{00}+1 \\
\mathcal{P} & \equiv(\mathbf{D} \times \mathbf{B}) \quad, \quad \mathcal{P}^{i}=T^{0 i}
\end{aligned}
$$

where $T^{\mu \nu}$ are components of symmetrical energy-momentum tensor

(see [6] where we designated it as $\bar{T}^{\mu \nu}$ ).

Then, from relations (2.2) we can obtain the following relation (see also [8, 9]):

$$
\left\{\begin{array}{l}
\mathbf{E}=\frac{1}{\alpha^{2}} \frac{\partial \mathcal{H}}{\partial \mathbf{D}}=\frac{1}{\mathcal{H}}\left(\mathbf{D}-\alpha^{2} \mathcal{P} \times \mathbf{B}\right)=\frac{1}{\mathcal{H}}\left[\left(1+\alpha^{2} \mathbf{B}^{2}\right) \mathbf{D}-\alpha^{2}(\mathbf{D} \cdot \mathbf{B}) \mathbf{B}\right] \\
\mathbf{H}=\frac{1}{\alpha^{2}} \frac{\partial \mathcal{H}}{\partial \mathbf{B}}=\frac{1}{\mathcal{H}}\left(\mathbf{B}+\alpha^{2} \mathcal{P} \times \mathbf{D}\right)=\frac{1}{\mathcal{H}}\left[\left(1+\alpha^{2} \mathbf{D}^{2}\right) \mathbf{B}-\alpha^{2}(\mathbf{D} \cdot \mathbf{B}) \mathbf{D}\right]
\end{array},\right.
$$

where

$$
\begin{aligned}
\mathcal{H} & =\sqrt{1+\alpha^{2}\left(\mathbf{D}^{2}+\mathbf{B}^{2}\right)+\alpha^{4} \mathcal{P}^{2}}= \\
& =\sqrt{\left(1+\alpha^{2} \mathbf{D}^{2}\right)\left(1+\alpha^{2} \mathbf{B}^{2}\right)-\alpha^{4}(\mathbf{D} \cdot \mathbf{B})^{2}} .
\end{aligned}
$$

Using relations (2.6) we can consider system (2.1) as the system of equations for fields D, B. This representation is best suitable for investigation of the singular dyon solutions.

Let us introduce the two electromagnetic potentials $A_{\mu}, \bar{A}_{\mu}$. In our case the potentials have singular line for each singular charged point (in three-dimensional 
space). We must exclude such line from space when consider any ordinary differential field model. The alternative way is connected with using distributions or generalized functions. As described by P.A.M. Dirac for monopoles [10], in this case we must include some distributions into definitions of the potentials through derivatives. But here we shall adhere to the first way associated with exclusion of the singular lines. Note that the singular currents into equations (2.1) set boundary conditions at the singular points (see [6]). In contrast, we shall take natural boundary conditions at the singular lines outside of the singular points.

Thus we define the potentials outside of any singular set with help the following formulas:

$$
F_{\mu \nu}=\partial_{\mu} A_{\nu}-\partial_{\nu} A_{\mu} \quad, \quad f^{\mu \nu}=-\varepsilon^{\mu \nu \sigma \rho} \partial_{\sigma} \bar{A}_{\rho}
$$

where $\varepsilon_{0123}=|g|^{1 / 2}, \quad \varepsilon^{0123}=-|g|^{-1 / 2}$, and

$$
\left\{\begin{array}{rl}
E_{i} & \equiv F_{i 0} \\
B^{i} & \equiv \frac{1}{2} \varepsilon^{i j k} F_{j k} \\
F_{i j} & =\varepsilon_{i j k} B^{k}
\end{array} \quad, \quad\left\{\begin{aligned}
D^{i} & \equiv f^{0 i} \\
H_{i} & \equiv \frac{1}{2} \varepsilon_{i j k} f^{j k} \\
f^{i j} & =\varepsilon^{i j k} H_{k}
\end{aligned}\right.\right.
$$

where $\varepsilon_{123}=|g|^{1 / 2}, \varepsilon^{123}=|g|^{-1 / 2},\left(\left|g_{00}\right|=1, g_{0 i}=0\right)$.

We have the following definition in three-dimensional designations:

$$
\begin{aligned}
& \left\{\begin{array}{l}
\mathbf{B}=\boldsymbol{\nabla} \times \mathbf{A} \\
\mathbf{D}=\boldsymbol{\nabla} \times \overline{\mathbf{A}}
\end{array}\right. \\
& \left\{\begin{array}{l}
\mathbf{H}=-\boldsymbol{\nabla} \bar{A}_{0}+\partial_{0} \overline{\mathbf{A}} \\
\mathbf{E}=\nabla A_{0}-\partial_{0} \mathbf{A}
\end{array}\right.
\end{aligned}
$$

From the basic equation of the model outside of the singularities [6]

$$
\frac{1}{\sqrt{|g|}} \frac{\partial}{\partial x^{\mu}} \sqrt{|g|} f^{\mu \nu}=0 \quad, \quad f^{\mu \nu}=\frac{1}{\mathcal{L}}\left(F^{\mu \nu}-\frac{\alpha^{2}}{2} \mathcal{J} \varepsilon^{\mu \nu \sigma \rho} F_{\sigma \rho}\right)
$$

we can easy obtain also the following equation for the potential $A_{\mu}$ outside of the singular set:

$$
C^{\mu \nu \sigma \rho} \frac{\partial^{2} A_{\rho}}{\partial x^{\mu} \partial x^{\sigma}}+\frac{\mathcal{L}}{2} f^{\mu \nu} \frac{\partial \ln |g|}{\partial x^{\mu}}=0
$$

where

$$
\begin{aligned}
C^{\mu \nu \sigma \rho} & =g^{\mu \sigma} g^{\nu \rho}-g^{\mu \rho} g^{\nu \sigma}-\alpha^{2}\left(\mathcal{F}^{\mu \nu} \mathcal{F}^{\sigma \rho}+f^{\mu \nu} f^{\sigma \rho}\right) \\
\mathcal{F}^{\mu \nu} & \equiv-\frac{1}{2} \varepsilon^{\mu \nu \alpha \rho} F_{\alpha \rho} .
\end{aligned}
$$




\section{Boundary conditions at the singular points}

Let us integrate system of equations (2.1) over a small four-dimensional space region including only $n$-th singular point. Using the partial integration in four-dimensional space we obtain the following conditions:

$$
\int_{-\infty}^{\infty}\left[\int_{\substack{n \\ \sigma}} f^{\mu \nu} \mathrm{d} \sigma_{\nu}^{n}-4 \pi \int_{\substack{n \\ \Omega}} j^{\mu} \mathrm{d} \Omega\right] \mathrm{d} x^{0}=0, \int_{-\infty}^{\infty}\left[\int_{\substack{n \\ \sigma}} \mathcal{F}^{\mu \nu} \mathrm{d} \sigma_{\nu}^{n}-4 \pi \int_{\substack{n \\ j^{\prime}}} \bar{\jmath}^{\mu} \mathrm{d} \Omega\right] \mathrm{d} x^{0}=0,
$$

where $\stackrel{n}{\Omega}$ is a three-dimensional region including only $n$-th singular point, $\mathrm{d} \boldsymbol{\sigma}$ is external directed element of the closed (two-dimensional) surface $\stackrel{n}{\sigma}$ enclosing the singular point (see also [6]),

$$
\begin{aligned}
& \mathrm{d} \Omega \equiv \sqrt{|g|}(\mathrm{d} x)^{3} \quad, \quad \mathrm{~d} \sigma_{0} \equiv-\stackrel{n}{\mathbf{V}} \cdot \mathrm{d} \stackrel{n}{\boldsymbol{\sigma}}, \\
& \mathrm{d} \sigma_{\mu} \mathrm{d} x^{0}=-\frac{1}{6} \varepsilon_{\mu \nu \sigma \rho} \mathrm{d} x^{\nu} \wedge \mathrm{d} x^{\sigma} \wedge \mathrm{d} x^{\rho} \text { are components of four-vector. }
\end{aligned}
$$

The surface $\stackrel{n}{\sigma}$ is rigidly coupled with $n$-th singular point and move with it together.

Relations (3.1) will be satisfied if we have in three-dimensional designations

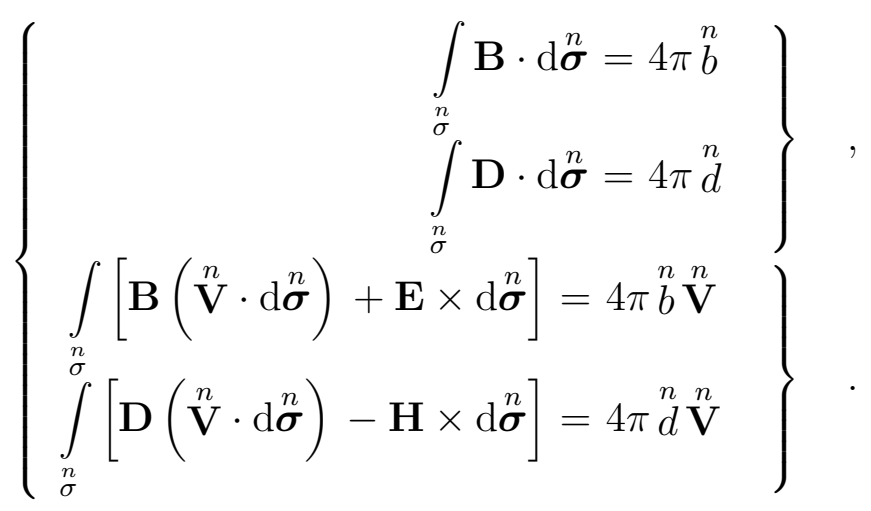

Because the surface $\stackrel{n}{\sigma}$ may be arbitrarily small, relations (3.2) are boundary conditions at the $n$-th singular point.

There is the attractive idea, proposed by A.S. Eddington [5], about an invariance of theory under permutation of points of space. We can apply this idea to the singular points. Let the model be invariant under permutation of any two singular points in the sense of change their charges $\stackrel{n_{1}}{d} \leftrightarrow \stackrel{n_{2}}{d}, \stackrel{n_{1}}{b} \leftrightarrow \stackrel{n_{2}}{b}$. Because the theory is invariant under change of charge's sign, in this case we have for any singular point

$$
\stackrel{n}{d}= \pm \bar{d} \quad, \quad \stackrel{n}{b}= \pm \bar{b},
$$

where $\bar{d}$ and $\bar{b}$ are some positive constants of space.

Thus we have the theory with three dimensional constants $\alpha, \bar{d}$ and $\bar{b}$. For the suitable dimensional system we can take $\alpha=1$ and $\bar{d}=1$ or $\bar{b}=1$. Thus we have the relation $\bar{d} / \bar{b}$ as the single dimensionless constant of the theory. Below (in section 9) we shall connect this relation with the fine structure constant. 


\section{Dyon solutions and singularities}

System of equations (2.1) with relations (2.6) has the following exact dyon solution in a cartesian coordinate system $\left\{y^{\mu}\right\}$ :

$$
\begin{array}{ll}
\underline{D}^{i}=\frac{y^{i} d}{r^{3}}, \quad \underline{B}^{i}=\frac{y^{i} b}{r^{3}}, \\
\underline{E}^{i}=\frac{y^{i} d}{r \sqrt{\bar{r}^{4}+r^{4}}} \quad, \quad \underline{H}^{i}=\frac{y^{i} b}{r \sqrt{\bar{r}^{4}+r^{4}}}, \\
\underline{A}_{0}=d \phi_{0}(r) \\
\underline{\bar{A}}_{0}=-b \phi_{0}(r) \quad, \quad \underline{\underline{\mathbf{A}}}=b \boldsymbol{\phi}(\mathbf{y}),
\end{array}
$$

where $\quad b= \pm \bar{b} \quad, \quad d= \pm \bar{d} \quad$

$$
r=\sqrt{y^{i} y_{i}} \quad, \quad \phi_{0}(r)=\int_{\infty}^{r} \frac{\mathrm{d} r^{\prime}}{\sqrt{\bar{r}^{4}+r^{\prime 4}}} \quad, \quad \bar{r} \equiv\left[\alpha^{2}\left(\bar{d}^{2}+\bar{b}^{2}\right)\right]^{\frac{1}{4}} .
$$

The vector function $\phi(\mathbf{y})$ may be of two types: with infinite singular line and with semi-infinite one. Its components have the following forms:

$$
\phi_{1}=\frac{1}{r} \frac{y^{2} y^{3}}{\rho^{2}}, \phi_{2}=-\frac{1}{r} \frac{y^{1} y^{3}}{\rho^{2}}, \phi_{3}=0
$$

or

$$
\phi_{1}=\frac{1}{r} \frac{\left(r+y^{3}\right) y^{2}}{\rho^{2}}, \quad \phi_{2}=-\frac{1}{r} \frac{\left(r+y^{3}\right) y^{1}}{\rho^{2}}, \quad \phi_{3}=0
$$

where $\quad \rho=\sqrt{\left(y^{1}\right)^{2}+\left(y^{2}\right)^{2}}$.

Function (4.3a) has the infinite singular line coinciding with the axis $y^{3}$ and functions $(4.3 \mathrm{~b})$ has the semi-infinite singular line in positive direction of $y^{3}$. In a spherical coordinate system $\{r, \vartheta, \varphi\}$ the both functions $\phi(\mathbf{y})$ have non-zero $\varphi$-components only that can be written in the forms

$$
\begin{array}{ccc}
(a) & \text { or } & (b) \\
\phi_{\varphi}=-\frac{\cot \vartheta}{r} & & \phi_{\varphi}=-\frac{1}{r} \cot \frac{\vartheta}{2}
\end{array}
$$

accordingly.

At the singular line the potentials $\mathbf{A}, \overline{\mathbf{A}}$ are devoid of defined direction and their absolute values are infinity. At the singular point $r=0$ the vectors $\mathbf{E}, \mathbf{H}, \mathbf{D}, \mathbf{B}$ are devoid of defined direction and absolute values of $\mathbf{D}, \mathbf{B}$ are infinity.

The full electromagnetic energy (see (6.3) below) of dyon solution (4.1) is

$$
\begin{aligned}
\overline{\mathcal{E}}= & \frac{2}{3}\left(\alpha^{-2} \beta \bar{r}^{3}\right), \\
& \text { where } \beta \equiv \int_{0}^{\infty} \frac{\mathrm{d} r}{\sqrt{1+r^{4}}}=\frac{\left[\Gamma\left(\frac{1}{4}\right)\right]^{2}}{4 \sqrt{\pi}} \approx 1.8541 .
\end{aligned}
$$


The dyon's energy has a space localization region. Let us denote the sphereenclosed dyon's energy as $\mathcal{E}^{\prime}$ for radius of the sphere $r^{\prime}$. Center of the sphere is at the origin of the coordinates $\left\{y^{i}\right\}$. The numerical calculation gives that $\mathcal{E}^{\prime}=0.5 \overline{\mathcal{E}}$ for $r^{\prime}=\bar{r}$ and $\mathcal{E}^{\prime}=0.95 \overline{\mathcal{E}}$ for $r^{\prime}=10 \bar{r}$.

With help of Lorentz transformation, shift, and rotation of the coordinate system $\left\{y^{i}\right\}$ we can obtain the following moving dyon solution in the coordinates $\left\{x^{\mu}\right\}$ :

$$
\begin{aligned}
& \left\{\begin{array}{l}
D^{i}=\left(\bar{L}_{j}^{i} \underline{D}^{j}-\varepsilon^{i j l} V_{j} \underline{H}_{l}\right) / \sqrt{1-\mathbf{V}^{2}} \\
B^{i}=\left(\bar{L}_{j}^{i} \underline{B}^{j}+\varepsilon^{i j l} V_{j} \underline{E}_{l}\right) / \sqrt{1-\mathbf{V}^{2}}
\end{array}\right. \\
& \left\{\begin{array}{l}
E^{i}=\left(\bar{L}_{j}^{i} \underline{E}^{j}-\varepsilon^{i j l} V_{j} \underline{B}_{l}\right) / \sqrt{1-\mathbf{V}^{2}} \\
H^{i}=\left(\bar{L}_{j}^{i} \underline{H}^{j}+\varepsilon^{i j l} V_{j} \underline{D}_{l}\right) / \sqrt{1-\mathbf{V}^{2}}
\end{array}\right. \\
& \left\{\begin{array}{l}
A_{\mu}=L_{. \mu}^{0} \underline{A}_{0}+L_{. \mu}^{i} \mathcal{R}_{. i}^{j} \underline{A}_{j}(\mathbf{z}) \\
\bar{A}_{\mu}=L_{. \mu}^{0} \underline{A}_{0}+L_{. \mu}^{i} \mathcal{R}_{. i}^{j} \underline{A}_{j}(\mathbf{z})
\end{array}\right.
\end{aligned}
$$

where $\underline{D}^{i}, \underline{B}^{i}, \underline{E}^{i}, \underline{H}^{i}, \underline{A}_{\nu}, \underline{\bar{A}}_{\nu}$ are defined by formulas (4.1) with $y^{i}=L_{j}^{i}\left(x^{j}-V^{j} x^{0}-a_{0}^{j}\right)$ and $z^{i}=\mathcal{R}_{. j}^{i} y^{j}$, $L_{. \mu}^{\nu}$ is the Lorentz transformation matrix, $L_{j}^{i} \bar{L}_{l}^{j}=\delta_{l}^{i}$,

$$
\begin{aligned}
& L_{.0}^{0}=\frac{1}{\sqrt{1-\mathrm{V}^{2}}}, \quad L_{. i}^{0}=L_{i 0}=-\frac{V_{i}}{\sqrt{1-\mathrm{V}^{2}}}, \\
& L_{j}^{i}=\delta_{j}^{i}+\left(\frac{1}{\sqrt{1-\mathrm{V}^{2}}}-1\right) \frac{V^{i} V_{j}}{\mathbf{V}^{2}}, \quad \bar{L}_{j}^{i}=\delta_{j}^{i}+\left(\sqrt{1-\mathrm{V}^{2}}-1\right) \frac{V^{i} V_{j}}{\mathbf{V}^{2}},
\end{aligned}
$$

$a_{0}^{j}$ are components of an initial position, $\mathcal{R}_{. j}^{i}$ is a rotation matrix.

\section{Variational principle for two potentials}

Here we propose some action such that the associated variational principle gives system of equations (2.1) with relations (2.6) and boundary conditions (3.2). This action has the following form:

$$
\begin{aligned}
\underline{S}=\int\left[(\underline{\mathcal{L}}+1)+2 \pi \alpha^{2}\left(\jmath^{\mu} A_{\mu}-\bar{\jmath}^{\mu} \bar{A}_{\mu}\right)\right] \sqrt{g}(\mathrm{~d} x)^{4} \\
\text { where } \quad \underline{\mathcal{L}} \equiv-\mathcal{H}+\frac{\alpha^{2}}{2}(\mathbf{E} \cdot \mathbf{D}+\mathbf{H} \cdot \mathbf{B}) \\
\mathcal{H}=\mathcal{H}(\mathbf{D}, \mathbf{B}) \text { according to (2.7), } \\
\mathbf{E}, \mathbf{B} \text { are represented by } A_{\mu} \text { and } \mathbf{D}, \mathbf{H} \text { are represented by } \bar{A}_{\mu} \text { with (2.10). }
\end{aligned}
$$

Using definition (2.5) we can easy write also the following expression for $\mathcal{L}$ :

$$
\underline{\mathcal{L}}=-\frac{1}{2}(\mathcal{L}+\overline{\mathcal{L}})
$$


Thus the action $\underline{S}$ is invariant under general transformation of coordinates.

If we substitute expression (5.3) for $\underline{\mathcal{L}}$ into the action $\underline{S}$ and take into account that $\mathcal{L}=\mathcal{L}\left(\partial_{\mu} A_{\nu}\right), \overline{\mathcal{L}}=\overline{\mathcal{L}}\left(\partial_{\mu} \bar{A}_{\nu}\right)$, then independent formal variation of eight components of the potentials $A_{\mu}, \bar{A}_{\nu}$ for the principle $\delta \underline{S}=0$ gives the following eight equations:

$$
\frac{1}{\sqrt{|g|}} \frac{\partial}{\partial x^{\mu}}\left[\sqrt{|g|} \frac{\partial \mathcal{L}}{\partial\left(\partial_{\nu} A_{\mu}\right)}\right]=4 \pi \jmath^{\nu}, \quad \frac{1}{\sqrt{|g|}} \frac{\partial}{\partial x^{\mu}}\left[\sqrt{|g|} \frac{\partial \overline{\mathcal{L}}}{\partial\left(\partial_{\nu} \bar{A}_{\mu}\right)}\right]=-4 \pi \bar{\jmath}^{\nu} .
$$

For simplicity we didn't consider here the singular lines of the potentials.

Formally we can consider system of equations (2.1) with relations (2.2) as the system of eight equations for the eight unknown functions $\left.A_{\mu}, \bar{A}_{\nu}\left(2.1 a^{\prime}\right), 2.1 b^{\prime}\right)$ for $\bar{A}_{\mu}$ and $\left(2.1 a^{\prime \prime}\right),\left(2.1 b^{\prime \prime}\right)$ for $\left.A_{\mu}\right)$. Such system of equations agree with system (5.4).

Now let us consider the action $\underline{S}$ (5.1) with the function $\underline{\mathcal{L}}$ in form (5.2). In this case the dependence of Lagrangian on the derivatives of potentials differs from one that we have for the function $\underline{\mathcal{L}}$ in form (5.3). Thus the appropriate variational principles with the function $\mathcal{L}$ in forms $(5.2)$ and $(5.3)$ are different.

Substituting definitions (2.10), (2.3), (2.4) into (5.1) we obtain the following expression for the action that doesn't contain $\delta$-functions:

$$
\begin{aligned}
\underline{S}= & \int(\underline{\mathcal{L}}+1) \sqrt{|g|}(\mathrm{d} x)^{4}+ \\
& +2 \pi \alpha^{2} \sum_{n=1}^{N} \int\left\langle\stackrel{n}{d}\left(A_{0}+\stackrel{n}{\mathbf{V}} \cdot \mathbf{A}\right)-\stackrel{n}{b}\left(\bar{A}_{0}+\stackrel{n}{\mathbf{V}} \cdot \overline{\mathbf{A}}\right)\right\rangle_{n} \mathrm{~d} x^{0} \\
\underline{\mathcal{L}}= & -\mathcal{H}+\frac{\alpha^{2}}{2}\left[\left(\nabla A_{0}-\partial_{0} \mathbf{A}\right) \cdot(\boldsymbol{\nabla} \times \overline{\mathbf{A}})+\left(\partial_{0} \overline{\mathbf{A}}-\boldsymbol{\nabla} \bar{A}_{0}\right) \cdot(\boldsymbol{\nabla} \times \mathbf{A})\right] .
\end{aligned}
$$

Note, though potentials $A_{\mu}, \bar{A}_{\mu}$ (4.6) for the dyon solutions are infinite at the singular lines, its averaging at the singular points $\left\langle A_{\mu}\right\rangle_{n},\left\langle\bar{A}_{\mu}\right\rangle_{n}$ are finite. Thus the action $\underline{S}$ is finite for the dyon solution.

Because definition of the potentials (2.10) may be used only outside of the singular set, expression for $\mathcal{L}$ (5.6) is true only outside of the singular lines. Let us enclose all singular set in a multi-tuple connected surface $\bar{\sigma}$ with external (relative to the singularity) surface element $\mathrm{d} \overline{\boldsymbol{\sigma}}$. Let this surface be composed of parts of surfaces $\stackrel{n}{\sigma}$ for the $N$ segregated

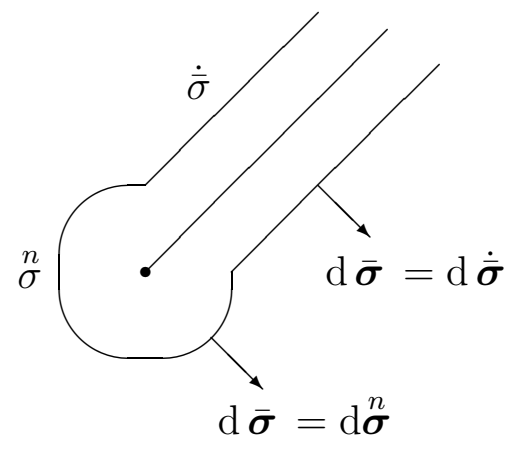
singular points and multi-tuply connected tubular surface $\dot{\bar{\sigma}}$ (in three-dimensional space) for parts of the singular lines outside neighbourhoods of the $N$ points. For 
example, the surface $\bar{\sigma}$ for the semi-infinite singular line can have image as it is shown in figure 1 .

Let us designate the action $\underline{S}$ in space outside of the singular set as $\underline{\bar{S}}$. We can obtain variation of this action with partial integration. Thus we have

$$
\begin{aligned}
& \delta \underline{\bar{S}}=\int_{-\infty}^{\infty} \mathrm{d} x^{0}\left\{\int_{\bar{\Omega}}\left[\left(\alpha^{2} \partial_{0} \mathbf{D}-\nabla \times \frac{\partial \mathcal{H}}{\partial \mathbf{B}}\right) \cdot \delta \mathbf{A}-\left(\alpha^{2} \partial_{0} \mathbf{B}+\nabla \times \frac{\partial \mathcal{H}}{\partial \mathbf{D}}\right) \cdot \delta \overline{\mathbf{A}}\right] \mathrm{d} \Omega\right. \\
& -\frac{\alpha^{2}}{2} \int_{\bar{\sigma}}\left[(\mathbf{D} \cdot \mathrm{d} \overline{\boldsymbol{\sigma}}) \delta A_{0}-(\mathbf{B} \cdot \mathrm{d} \overline{\boldsymbol{\sigma}}) \delta \bar{A}_{0}\right]+2 \pi \alpha^{2} \sum_{n}\left(\stackrel{n}{d}\left\langle\delta A_{0}\right\rangle_{n}-\stackrel{n}{b}\left\langle\delta \bar{A}_{0}\right\rangle_{n}\right) \\
& +\int_{\bar{\sigma}}\left[\frac{\partial \mathcal{H}}{\partial \mathbf{D}} \times \mathrm{d} \overline{\boldsymbol{\sigma}}-\frac{\alpha^{2}}{2}\left(\mathbf{E} \times \mathrm{d} \overline{\boldsymbol{\sigma}}+\mathbf{B} \mathrm{d} \bar{\sigma}_{0}\right)\right] \cdot \delta \overline{\mathbf{A}}-2 \pi \alpha^{2} \sum_{n}^{n} b \stackrel{n}{\mathbf{V}} \cdot\langle\delta \overline{\mathbf{A}}\rangle_{n} \\
& +\int_{\bar{\sigma}}\left[\frac{\partial \mathcal{H}}{\partial \mathbf{B}} \times \mathrm{d} \overline{\boldsymbol{\sigma}}-\frac{\alpha^{2}}{2}\left(\mathbf{H} \times \mathrm{d} \overline{\boldsymbol{\sigma}}-\mathbf{D} \mathrm{d} \bar{\sigma}_{0}\right)\right] \cdot \delta \mathbf{A}+2 \pi \alpha^{2} \sum_{n}^{n} \stackrel{n}{\mathrm{~V}} \cdot\langle\delta \mathbf{A}\rangle_{n} \\
& \left.+2 \pi \alpha^{2} \sum_{n}\left[\langle\stackrel{n}{d}(\mathbf{E}+\stackrel{n}{\mathbf{V}} \times \mathbf{B})+\stackrel{n}{b}(\mathbf{H}-\stackrel{n}{\mathbf{V}} \times \mathbf{D})\rangle_{n} \cdot \delta \mathbf{a}\right]-\int_{\bar{\sigma}} \underline{\mathcal{L}} \mathrm{d} \overline{\boldsymbol{\sigma}} \cdot \delta \delta^{\bar{\sigma}}\right\}
\end{aligned}
$$

where $\bar{\Omega}$ is three-dimensional space without the region bounded by surface $\bar{\sigma}$,

$$
\mathrm{d} \bar{\sigma}_{0} \equiv-(\stackrel{\bar{\sigma}}{\mathbf{V}} \cdot \mathrm{d} \overline{\boldsymbol{\sigma}}), \quad \stackrel{\bar{\sigma}}{\mathbf{V}} \equiv \frac{\mathrm{d}}{\mathrm{d} x^{0}}, \quad \stackrel{\overline{\mathbf{a}}}{\mathbf{a}} \text { is a points of the surface } \bar{\sigma} .
$$

Now we contract the surface $\dot{\bar{\sigma}}$ to the singular lines outside neighbourhoods of the $N$ singular points. Next we contract the surfaces $\stackrel{n}{\sigma}$ to the $N$ singular points. As result we have $\delta \underline{\bar{S}} \rightarrow \delta \underline{S}$.

Let us find stationary conditions for the action $\underline{S}$. By the general principle of calculus of variations [1], we can take $\delta_{\mathbf{a}}^{n}=\delta_{\mathbf{a}}^{\bar{a}}=0$ at first. We can also take continuous variations $\delta A_{\mu}, \delta \bar{A}_{\mu}$ and make, firstly, $\delta A_{\mu}=0, \delta \bar{A}_{\mu}=0$ at the singular lines. Then, according to the first line in expression (5.7), we have

$$
\alpha^{2} \partial_{0} \mathbf{B}+\nabla \times \frac{\partial \mathcal{H}}{\partial \mathbf{D}}=0 \quad, \quad \alpha^{2} \partial_{0} \mathbf{D}-\nabla \times \frac{\partial \mathcal{H}}{\partial \mathbf{B}}=0
$$

in the space outside of the singular lines. Using definition of the fields $\mathbf{B}, \mathbf{D}, \mathbf{E}, \mathbf{H}$ through potentials (2.10), we obtain from equations (5.8) that

$$
\mathbf{E}=\frac{1}{\alpha^{2}} \frac{\partial \mathcal{H}}{\partial \mathbf{D}} \quad, \quad \mathbf{H}=\frac{1}{\alpha^{2}} \frac{\partial \mathcal{H}}{\partial \mathbf{B}} .
$$

Now we take $\delta A_{\mu} \neq 0$ and $\delta \bar{A}_{\mu} \neq 0$ at the singular lines for continuous variations $\delta A_{\mu}, \delta \bar{A}_{\mu}$. We assume that the fields $\mathbf{D}, \mathbf{B}, \mathbf{E}, \mathbf{H}$ are continuous at the singular lines outside the segregated singular points. Then we obtain boundary conditions (3.2) from expression (5.7) (line 2-4), using formulas (5.9). 
Finally, we take $\delta \mathbf{a}^{n} \neq 0, \delta \delta_{\mathbf{a}}^{\bar{a}} \neq 0$. It would appear reasonable that at the singular lines

$$
\lim _{\bar{\sigma} \rightarrow 0} \int_{\bar{\sigma}} \underline{\mathcal{L}} \mathrm{d} \overline{\boldsymbol{\sigma}}=0
$$

Then, from the last line in (5.7) we have the following condition at the $n$-th singular point:

$$
\langle\stackrel{n}{d}(\mathbf{E}+\stackrel{n}{\mathbf{V}} \times \mathbf{B})+\stackrel{n}{b}(\mathbf{H}-\stackrel{n}{\mathbf{V}} \times \mathbf{D})\rangle_{n}=0
$$

This condition generalize one for purely electrical singularity $(\stackrel{n}{b}=0)$, which had obtained in the article [6], to the case of dyon singular point. Using definition of singular currents (2.3) we obtain also the following form of the condition:

$$
F_{\mu \nu} \jmath^{\nu}-\frac{1}{2} \varepsilon_{\mu \nu \sigma \rho} f^{\sigma \rho} \bar{\jmath}^{\nu}=0
$$

Thus the variational principle for action $\underline{S}$ (5.1), (5.5) gives system of equations (2.1) and point boundary conditions (3.2), (5.11).

Notice that here we don't use a concrete form for the function $\mathcal{H}(\mathbf{D}, \mathbf{B})$. Thus this derivation is suitable for any electrodynamic models.

\section{Conservation laws and comments about dimensions}

Using equations (2.1) with formulas (2.6) and condition (5.12) we can check directly the following conservation law for symmetrical energy-momentum tensor (in cartesian coordinates):

$$
\frac{\partial T^{\mu \nu}}{\partial x^{\mu}}=0
$$

where

$$
\begin{aligned}
& T^{00}=\alpha^{-2}(\mathcal{H}-1), \\
& T^{0 i}=\varepsilon^{i j l} D_{j} B_{l}=\varepsilon^{i j l} E_{j} H_{l}, \\
& T^{i j}=\delta^{i j}\left[\mathbf{D} \cdot \mathbf{E}+\mathbf{B} \cdot \mathbf{H}-T^{00}\right]-\left(D^{i} E^{j}+B^{i} H^{j}\right) .
\end{aligned}
$$

Let us introduce the following notations for full energy and momentum in the region $\tilde{\Omega}$ of three-dimensional space $\Omega$.

$$
\mathcal{E}=\frac{1}{4 \pi \alpha^{2}} \int_{\tilde{\Omega}}(\mathcal{H}-1) \mathrm{d} \Omega \quad, \quad \mathbf{P}=\frac{1}{4 \pi} \int_{\tilde{\Omega}} \mathcal{P} \mathrm{d} \Omega
$$

where $\mathcal{P}^{i} \equiv T^{0 i}$ or $\mathcal{P} \equiv \mathbf{D} \times \mathbf{B}$. 
Let the region $\tilde{\Omega}$ may be moving, that is we have $\tilde{\Omega}=\tilde{\Omega}\left(x^{0}\right)$. Then, by integration of conservation law (6.1) over the region $\tilde{\Omega}$ we obtain the following integral conservation law for energy-momentum:

$$
\begin{aligned}
\frac{\mathrm{d} \mathcal{E}}{\mathrm{d} x^{0}} & =-\frac{1}{4 \pi} \int_{\tilde{\sigma}} \mathcal{P} \cdot \mathrm{d} \tilde{\boldsymbol{\sigma}}-\frac{1}{4 \pi \alpha^{2}} \int_{\tilde{\sigma}}(\mathcal{H}-1) \mathrm{d} \tilde{\sigma}_{0}, \\
\frac{\mathrm{d} P^{i}}{\mathrm{~d} x^{0}} & =-\frac{1}{4 \pi} \int_{\tilde{\sigma}} T^{i j} \mathrm{~d} \tilde{\sigma}_{j}-\frac{1}{4 \pi} \int_{\tilde{\sigma}} \mathcal{P}^{i} \mathrm{~d} \tilde{\sigma}_{0},
\end{aligned}
$$

where $\tilde{\sigma}$ is the surface that bounds the region $\tilde{\Omega}$,

$\mathrm{d} \tilde{\sigma}_{j}$ is the external element of this surface,

$\mathrm{d} \tilde{\sigma}_{0} \equiv-\tilde{\mathbf{V}} \cdot \mathrm{d} \tilde{\boldsymbol{\sigma}}, \quad \tilde{\mathbf{V}}$ is the velocity of surface's point.

It is evident, from (6.1) we have also the following conservation law for angular momentum tensor:

$$
\frac{\partial M^{\mu \nu \rho}}{\partial x^{\mu}}=0
$$

where

$$
M^{\mu \nu \rho}=T^{\mu \nu} x^{\rho}-T^{\mu \rho} x^{\nu}
$$

We introduce the following notation for vector of full angular momentum in the region $\tilde{\Omega}$ :

$$
\mathbf{M}=\frac{1}{4 \pi} \int_{\tilde{\Omega}}(\mathcal{P} \times \mathbf{x}) \mathrm{d} \Omega
$$

Let us take up somewhat the dimensions of the quantities. Because we don't explicitly use the speed of light $c$, the temporary coordinate $x^{0}$ has a dimension identical to that for the space coordinates $x^{i}$, that is dimension of length. (But we can make substitution $x^{0} \rightarrow c t$ for all time.) For the same reason the momentum has a dimension identical to that for the energy. And dimension of the full angular momentum is $[M]=[P][x]=[\mathcal{E}][x]$. In this case the dimension of mass is identical to that for the energy.

\section{Method for investigation of interaction between dyons}

We shall consider system (2.1) with relations (2.6) as system of equations for the fields D, B. Let us write this system in the following formal operational form:

$$
\mathcal{N} \mathcal{D}=0 \quad, \quad \text { where } \quad \mathcal{D} \equiv\left(\begin{array}{l}
\mathbf{D} \\
\mathbf{B}
\end{array}\right)
$$


We can search a solution of equation (7.1) in the form of the following formal series:

$$
\mathcal{D}=\mathcal{D}^{(0)}+\mathcal{D}^{1)}+\mathcal{D}^{2)}+\ldots \quad, \quad \mathcal{D}^{(k)} \equiv \mathcal{D}^{(0)}+\sum_{l=1}^{k} \mathcal{D}^{l)}
$$

where $\mathcal{D}^{(0)}$ is an initial approximation,

$\mathcal{D}^{k)} \quad$ is $k$-th correction,

$\mathcal{D}^{(k)}$ is $k$-th approximation.

Let us expand the operator $N$ into equation (7.1) near to the $k$-th approximation $\mathcal{D}^{(k)}$ and keep only linear terms by the correction $\mathcal{D}^{k+1)}$. Thus we obtain the following equation for the correction:

$$
\left\{\mathcal{N}^{\prime}\left[\mathcal{D}^{(k)}\right]\right\} \mathcal{D}^{k+1)}=-\mathcal{N} \mathcal{D}^{(k)}
$$

where $\mathcal{N}^{\prime}$ is Frechet's derivative for the operator $\mathcal{N}$.

Equation (7.3) gives the following iterative process, which is called Newton's method for operators [12]:

$$
\mathcal{D}^{(k+1)}=\mathcal{D}^{(k)}-\left\{\mathcal{N}^{\prime}\left[\mathcal{D}^{(k)}\right]\right\}^{-1}\left[\mathcal{N} \mathcal{D}^{(k)}\right] .
$$

An alternative possible way is based on using the principle of contractive mappings. Let us rewrite system (7.1) in the following form:

$$
\mathcal{M D}=\tilde{\mathcal{N}} \mathcal{D} \quad, \quad \text { where } \quad \tilde{\mathcal{N}} \mathcal{D} \equiv \mathcal{M D}-\mathcal{N} \mathcal{D}
$$

and $\mathcal{M}$ is the operator represented by left parts of linear Maxwell equations for $\mathbf{D}=\mathbf{E}, \mathbf{B}=\mathbf{H}$

In this case we can easy obtain the appropriate inverse operator $\mathcal{M}^{-1}$. As result we have the following representation for equation (7.1):

$$
\mathcal{D}=\mathcal{M}^{-1} \tilde{\mathcal{N}} \mathcal{D}
$$

If the operator $\mathcal{M}^{-1} \tilde{\mathcal{N}}$ is contractive (for some given class of functions including the initial approximation), then the iterative process

$$
\mathcal{D}^{(k+1)}=\mathcal{M}^{-1} \tilde{\mathcal{N}} \mathcal{D}^{(k)}
$$

converges to the solution.

The convergence of iterative processes (7.4), (7.7) to some solution is defined by conditions that depend on initial approximation [12]. But we can use these procedures, even if it doesn't converge. In this case we may have some number of iteration for best approximation to the solution. In any case the initial approximation $\mathcal{D}^{(0)}$ must be sufficiently close to some exact solution. 
We can take the sum of some solutions as initial approximation. Let us take moving dyons $\stackrel{n}{\mathcal{D}}$ (4.6) as these solutions. Thus we have

$$
\mathcal{D}^{(0)}=\sum_{n}^{N} \stackrel{n}{\mathcal{D}}
$$

The field configuration $\mathbf{D}, \mathbf{B}$ for the exact moving dyon solution has six free parameters: three components of the velocity and three components of the initial position. We can assume that these parameters for the dyons into initial approximation (7.8) are functions of time. This is standard practice for similar problems. But, as it can show, if we take that the initial positions depend on time, then boundary conditions $(3.2 \mathrm{~b})$ are broken for the dyon field configuration. Thus we may have only three components of velocity as dyon's parameters depending on time.

We believe that each dyon has an optional trajectory $\mathbf{x}=\stackrel{n}{\mathbf{a}}\left(x^{0}\right)$ such that

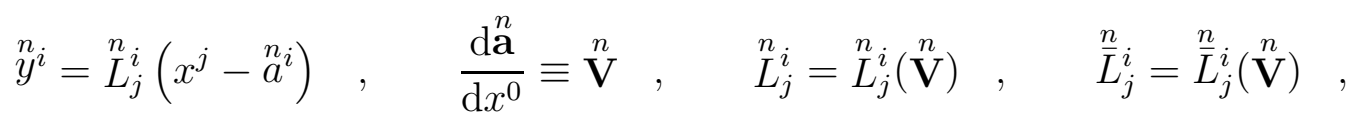

where $\stackrel{n^{i}}{y^{i}}$ are included into expressions (4.1 a), (4.1 b),

$$
\bar{L}_{j}^{i} \text { are included into expressions (4.6a). }
$$

The trajectories $\mathbf{a}\left(x^{0}\right)$ must be obtained.

In the same manner as for field we can represent the functions $\stackrel{n}{\mathbf{a}\left(x^{0}\right)}$ by the following series:

$$
\left.\stackrel{n}{\mathbf{a}}=\mathbf{a}^{n}(0)+\mathbf{a}^{n}\right)+\mathbf{a}^{n}+\ldots \quad, \quad \mathbf{a}^{n}(k) \equiv \mathbf{a}^{n}(0)+\sum_{l=1}^{k} \mathbf{a}^{l)},
$$

where $\stackrel{\mathbf{a}}{(0)}^{n}$ is the trajectory of $n$-th dyon for the initial approximation,

$\mathbf{a}^{n}{ }^{k} \quad$ is $k$-th correction to the trajectory,

$\mathbf{a}^{n}(k)$ is $k$-th approximation for the trajectory.

If we have obtained the trajectories in initial approximation $\mathbf{a}^{n}(0)\left(x^{0}\right)$ with help some method, then we can obtain the first correction $\mathcal{D}^{1)}$ as solution to equation (7.3) or from formula (7.7) for $k=0$. This correction $\mathcal{D}^{1)}$ is a radiation of the dyons accelerated according to their trajectories $\stackrel{\mathbf{a}}{(0)}^{(0)}\left(x^{0}\right)$. But the radiation of the dyons modifies their trajectories. We describe these modifications by functions $\mathbf{a}^{1)}$ that correct the trajectories $\mathbf{a}^{n}(0)$. Thus we must obtain the corrections $\stackrel{\mathbf{a}}{k}^{k)}$ to the trajectories $\mathbf{a}^{n}(k-1)$ in any step of iteration. In effect, this is a correction of the initial approximation (through the correction of the trajectories) for every iteration step.

A direct calculation gives that all boundary conditions (3.2), (5.11) are satisfied for initial field configuration (7.8) with (7.9) and optional trajectories of the dyons. This is distinction of this method from one that had used in the article [6], where we take the four components of the potential $A_{\mu}$ as unknown functions. But using 
the fields D, B as unknown functions, the present method is more suitable for satisfaction to all point boundary conditions in any iteration step.

In the present article we shall use integral energy-momentum conservation law (6.4) for obtaining trajectories of the interacting dyons.

Notice also the following. Because near $n$-th singular point absolute values of the fields $\stackrel{n}{\mathbf{D}}, \stackrel{n}{\mathbf{B}}$ tends to infinity, for any finite given external field (of other dyons) we can define some neighbourhood of the singular point, where this external field may be believed to be small. Thus the consideration of any (small) distances between the dyons in initial approximation may be worthwhile.

\section{Interaction of dyon with a small given field}

Let us consider the interaction of moving dyon with a small given field $\stackrel{n}{\tilde{\mathcal{D}}^{i}}$ $(i=1, \ldots, 6),(\stackrel{n}{\tilde{\mathbf{E}}} \approx \stackrel{n}{\sim} \mathbf{\mathbf { D }}, \stackrel{n}{\tilde{\mathbf{H}}} \approx \stackrel{n}{\tilde{\mathbf{B}}})$ in initial approximation. In particular, this small field may represent the field of other dyons that are at sufficiently long distance from the dyon under consideration.

In this case we consider the space region $\stackrel{n}{\Omega}$ that include the dyon under consideration only. The region $\stackrel{n}{\Omega}$ move with $n$-th singularity together. We can take a maximal absolute value of the functions $\stackrel{n}{\mathcal{D}}^{i}$ in the region $\stackrel{n}{\Omega}$ as the small order parameter $\epsilon$ for the problem of finding the first approximation in the region $\stackrel{n}{\widetilde{\Omega}}$. Here we consider the case when the region $\stackrel{n}{\Omega}$ has more size than one for dyon localization region (see section (四)).

Thus for the region $\stackrel{n}{\Omega}$ we take

$$
\mathcal{D}^{(0)}=\stackrel{n}{\mathcal{D}}+\stackrel{n}{\tilde{\mathcal{D}}}
$$

For obtaining dyon's trajectory equation we use formula (6.4 $\mathrm{b})$. We consider the case when the field $\stackrel{n}{\tilde{\mathcal{D}}}$ is approximately constant into the region $\stackrel{n}{\widetilde{\Omega}}$. Thus we have

$$
\frac{\mathrm{d} \mathbf{P}^{(0)}}{\mathrm{d} x^{0}}=\frac{\mathrm{d} \stackrel{n}{\mathbf{P}}}{\mathrm{d} x^{0}}=\frac{\mathrm{d}}{\mathrm{d} x^{0}} \frac{\overline{\mathcal{E}} \stackrel{n}{\mathbf{V}}}{\sqrt{1-\mathbf{V}^{2}}},
$$

where $\overline{\mathcal{E}}$ is defined into (4.5). Here we take into account that size of the region $\stackrel{n}{\widetilde{\Omega}}$ is much more than $\bar{r}(4.2)$.

Now we expand the components $T^{i j}$ as Taylor series near the $n$-th dyon's field configuration. We restrict the consideration to the terms of no more than the first order by $\epsilon$. Thus we have

$$
T^{i j}=\stackrel{n}{T}^{i j}+\frac{\partial \stackrel{n}{T}^{i j}}{\partial \mathcal{D}^{l}} \stackrel{n}{\mathcal{D}}^{l}+\ldots
$$


where $\quad l=1, \ldots, 6 \quad,\left.\quad \stackrel{n}{T^{i j}} \equiv T^{i j}\right|_{\mathcal{D}^{l}=\stackrel{n}{\mathcal{D}^{l}}} \quad,\left.\quad \frac{\partial \stackrel{n}{T}^{i j}}{\partial \mathcal{D}^{l}} \equiv \frac{\partial T^{i j}}{\partial \mathcal{D}^{l}}\right|_{\mathcal{D}^{l}=\mathcal{D}^{l}}$

According to formulas (6.2) we have

$$
\begin{aligned}
& \frac{\partial T^{i j}}{\partial D^{q}}=\frac{1}{\alpha^{2}}\left[-\delta_{q}^{i} \frac{\partial \mathcal{H}}{\partial D_{j}}+\left(\delta^{i j} \delta_{p}^{s}-\delta_{p}^{i} \delta^{j s}\right)\left(D^{p} \frac{\partial^{2} \mathcal{H}}{\partial D^{s} \partial D^{q}}+B^{p} \frac{\partial^{2} \mathcal{H}}{\partial B^{s} \partial D^{q}}\right)\right] \\
& \frac{\partial T^{i j}}{\partial B^{q}}=\frac{1}{\alpha^{2}}\left[-\delta_{q}^{i} \frac{\partial \mathcal{H}}{\partial B_{j}}+\left(\delta^{i j} \delta_{p}^{s}-\delta_{p}^{i} \delta^{j s}\right)\left(D^{p} \frac{\partial^{2} \mathcal{H}}{\partial D^{s} \partial B^{q}}+B^{p} \frac{\partial^{2} \mathcal{H}}{\partial B^{s} \partial B^{q}}\right)\right]
\end{aligned}
$$

We have also

$$
\begin{aligned}
& \frac{\partial^{2} \mathcal{H}}{\partial D^{i} \partial D^{j}}=\frac{\alpha^{2}}{\mathcal{H}}\left[\delta_{i j}\left(1+\alpha^{2} \mathbf{B}^{2}\right)-\alpha^{2}\left(B_{i} B_{j}+E_{i} E_{j}\right)\right] \\
& \frac{\partial^{2} \mathcal{H}}{\partial B^{i} \partial B^{j}}=\frac{\alpha^{2}}{\mathcal{H}}\left[\delta_{i j}\left(1+\alpha^{2} \mathbf{D}^{2}\right)-\alpha^{2}\left(D_{i} D_{j}+H_{i} H_{j}\right)\right] \\
& \frac{\partial^{2} \mathcal{H}}{\partial D^{i} \partial B^{j}}=\frac{\alpha^{4}}{\mathcal{H}}\left[2 D_{i} B_{j}-\delta_{i j}(\mathbf{D} \cdot \mathbf{B})-B_{i} D_{j}-E_{i} H_{j}\right]
\end{aligned}
$$

Using these formulas and formulas (2.6) we obtain from (8.4) that

$$
\begin{aligned}
& \frac{\partial \stackrel{n}{T}^{i j}}{\partial D^{q}}=-\delta_{q}^{i} \stackrel{n}{E} j+\delta^{i j} \stackrel{n}{E_{q}}-\delta_{q}^{j} \stackrel{n}{E}^{i}+O\left(1 / \stackrel{n}{r}^{4}\right), \\
& \frac{\partial \stackrel{n}{T^{i j}}}{\partial B^{q}}=-\delta_{q}^{i} \stackrel{n}{H}^{j}+\delta^{i j} \stackrel{n}{H} q-\delta_{q}^{j} \stackrel{n}{H}^{i}+O\left(1 / \stackrel{n}{r}^{4}\right) .
\end{aligned}
$$

We substitute (8.2), (8.3), (8.6) into relation (6.4 b) and make the integration for its right part. Because the symmetry properties of element of integration, the last integral is zero. As result we obtain the following generalized Lorentz equation for the trajectory of $n$-th dyon:

$$
\overline{\mathcal{E}} \frac{\mathrm{d}}{\mathrm{d} x^{0}} \frac{\stackrel{n}{\mathbf{V}}}{\sqrt{1-\mathbf{V}^{2}}}=\stackrel{n}{d}(\stackrel{n}{\tilde{\mathbf{D}}}+\stackrel{n}{\mathbf{V}} \times \stackrel{n}{\tilde{\mathbf{B}}})+\stackrel{n}{b}(\stackrel{n}{\tilde{\mathbf{B}}}-\stackrel{n}{\mathbf{V}} \times \stackrel{n}{\tilde{\mathbf{D}}})
$$

As we see, the energy-momentum method for obtaining the trajectories gives the value of mass which is equal to rest energy of the dyon. This result fully conform with Einstein's principle for equivalence of mass to energy.

In the article [6] we considered the potentials $A_{\mu}$ as unknown functions and used the boundary condition of type (5.11) for obtaining the trajectory equation. As result we had obtained another value of mass in initial approximation $\left(\frac{3}{2} \overline{\mathcal{E}}\right)$ than in the present article. Generally speaking, we have certain arbitrariness for taking an initial trajectory of the singularity. Because a true trajectory of the singularity is defined by an exact solution only, we can obtain corrections to the initial trajectory (in first and highest iteration steps) such that it will appear as renormalization of mass. But the method of the present article is more suitable, because it gives the satisfaction as energy-momentum conservation law (6.4) as boundary condition (5.11) for any iteration step. 


\section{Bidyon or an electromagnetic model of particle with spin}

Let us consider the interaction of two dyon singularities with identical electric and opposite magnetic charges: $\stackrel{1}{d}=\stackrel{2}{d}, \stackrel{1}{b}=-\stackrel{2}{b}$. At first we use a cylindrical coordinate system $\{z, \rho, \varphi\}$ such that dyons are on the axis $z$. We believe also that the dyons velocities are directed with the axis $z$ and the problem is symmetrical about the plane $z=0$. This configuration is shown in figure Q 2 , where $d= \pm \bar{d}, b= \pm \bar{b}$.

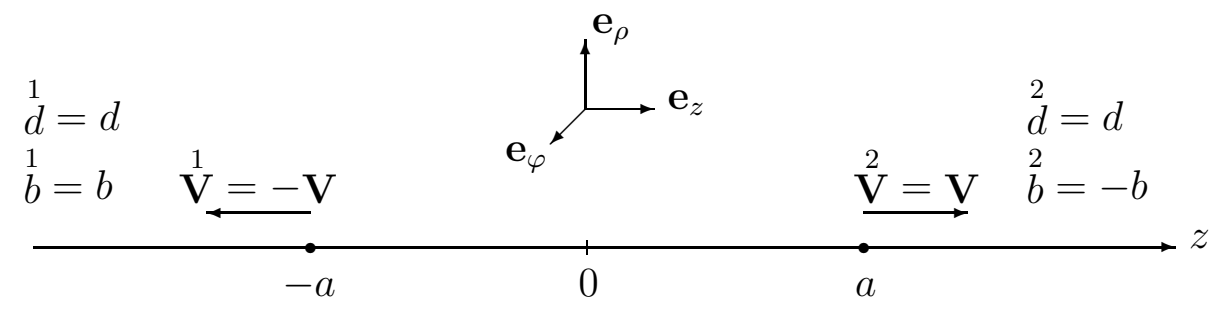

Figure 2: Disposition of the two dyons in the cylindrical coordinate system.

We name this disposition of the singularities with appropriate field configuration as bidyon.

According to our iterative method (see section (7) we take the sum of the fields for moving dyons (4.6) with unknown their trajectory $a\left(x^{0}\right)$ as initial approximation. That is we have

$$
\mathcal{D}^{(0)}=\stackrel{1}{\mathcal{D}}+\stackrel{2}{\mathcal{D}}
$$

In the cylindrical coordinate system we have the following expressions for the fields $\stackrel{1}{\mathbf{D}}, \stackrel{2}{\mathbf{D}}$ and $\stackrel{1}{\mathbf{B}}, \stackrel{2}{\mathbf{B}}$ :

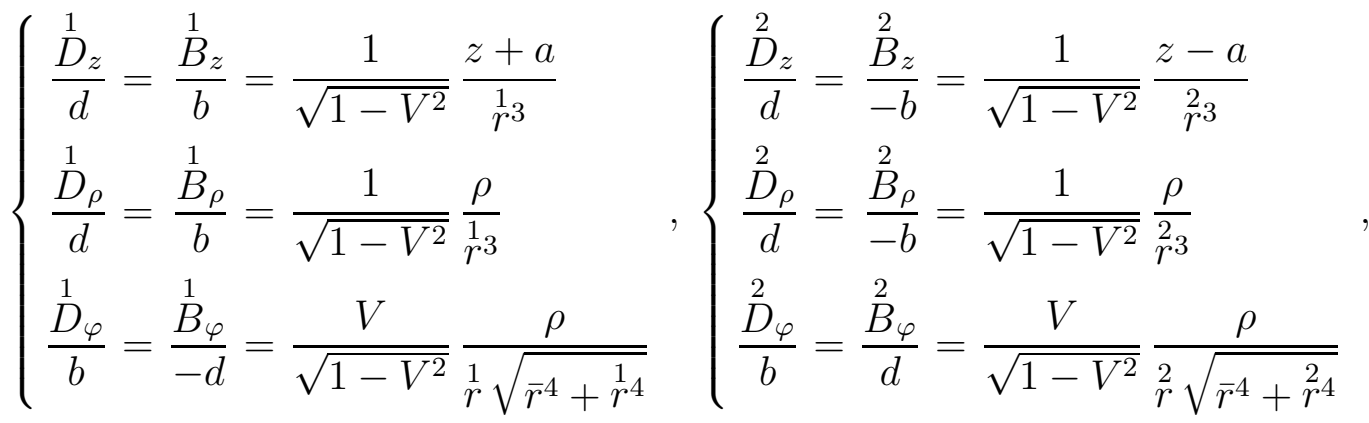

$$
\begin{aligned}
& \text { where } \quad V \equiv \frac{\mathrm{d} a}{\mathrm{~d} x^{0}}, \stackrel{1}{r}=\sqrt{\left(z^{\prime}+a^{\prime}\right)^{2}+\rho^{2}}, \quad \stackrel{2}{r}=\sqrt{\left(z^{\prime}-a^{\prime}\right)^{2}+\rho^{2}}, \\
& z^{\prime} \equiv \frac{z}{\sqrt{1-V^{2}}} \quad, \quad a^{\prime} \equiv \frac{a}{\sqrt{1-V^{2}}} \quad .
\end{aligned}
$$

The electromagnetic potentials don't use in our iterative method. But from the initial approximation we can make some suppositions about true field configuration of the potentials for the appropriate exact solution. It is reasonable to take that the 
both dyons into (9.1) have the singular lines coinciding with the axis $z$. That is we have

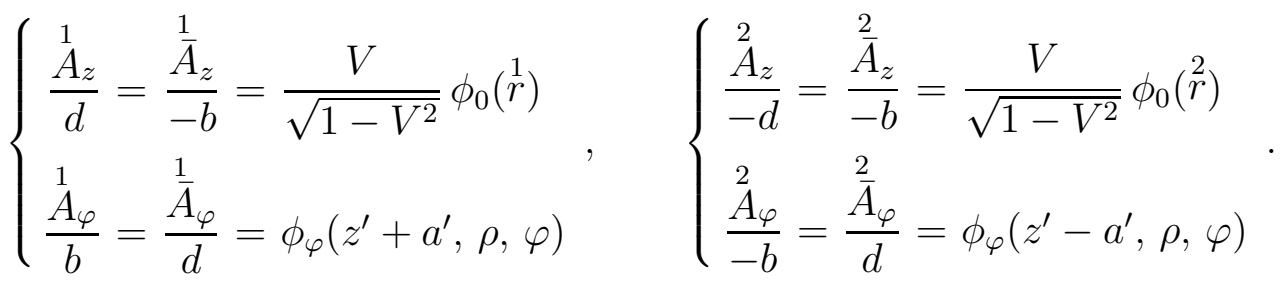

Here we may take the infinite singular lines with function $\phi_{\varphi}$ of type (4.4a). In this case the singular lines for the potential A particularly cancel each other outside of the interval between the dyons. This cancelling is complete as $z \rightarrow \pm \infty$ or $a \rightarrow 0$. If we also believe that at the singular points the field $\mathcal{D}$ of the bidyon solution are equal to the field for the dyon solution $\stackrel{1}{\mathcal{D}}$ or $\stackrel{2}{\mathcal{D}}$, we must believe that the exact solution has the potential $\mathbf{A}$ with the function $\phi_{\varphi}$ of type $(4.4 \mathrm{~b})$ at the singular points. Thus we can presume that the potential $\mathbf{A}$ for the associated exact solution has the singular line segment between the dyons only. That is we have a singular string segment (for $\mathbf{A}$ ) with the dyons at the ends.

Let us calculate the full angular momentum for field configuration (9.1). We use definition (6.7) for full three-dimensional space. Because of the symmetry property of elements of integration into (6.7), for this case we have $M_{\rho}=M_{\varphi}=0$,

$$
M_{z}=-\frac{1}{4 \pi} \int \mathcal{P}_{\varphi} \rho(\rho \mathrm{d} z \mathrm{~d} \rho \mathrm{d} \varphi)
$$

We can easy obtain the following expression:

$$
\mathcal{P}_{\varphi}=\frac{-4 a b d \rho}{r^{3} \stackrel{2}{r}^{3}\left(1-V^{2}\right)} .
$$

Now we introduce new variables of integration $\{\xi, \zeta, \varphi\}$ that appropriate to the bispherical coordinate system:

$$
z^{\prime}=\frac{a^{\prime} \sinh \xi}{\cosh \xi-\cos \zeta} \quad, \quad \rho=\frac{a^{\prime} \sin \zeta}{\cosh \xi-\cos \zeta} .
$$

We have the bispherical element of value

$$
\left(\rho \mathrm{d} z^{\prime} \mathrm{d} \rho \mathrm{d} \varphi\right)=\frac{a^{\prime 3} \sin \zeta \mathrm{d} \xi \mathrm{d} \zeta \mathrm{d} \varphi}{(\cosh \xi-\cos \zeta)^{3}}
$$

We have also the following expression for $\stackrel{1}{r}, \stackrel{2}{r}$ :

$$
\stackrel{1}{r}=\frac{\sqrt{2} a^{\prime} \exp (\xi / 2)}{\sqrt{\cosh \xi-\cos \zeta}} \quad, \quad \stackrel{2}{r}=\frac{\sqrt{2} a^{\prime} \exp (-\xi / 2)}{\sqrt{\cosh \xi-\cos \zeta}}
$$


Substituting (9.6), (9.8) into (9.5) and introducing the variable $\underline{z}=\cos \zeta$, we obtain the following expression:

$$
\mathcal{P}_{\varphi}=-b d \frac{\left(1-V^{2}\right)^{\frac{3}{2}}}{2 a^{4}}(\cosh \xi-\underline{z})^{2} \sqrt{1-\underline{z}^{2}} .
$$

And substituting (9.6), (9.7), (9.9) into (9.4) we obtain the following expression for $z$-component of the full angular momentum:

$$
M_{z}=\frac{b d}{8 \pi} \int_{-\pi}^{\pi} \mathrm{d} \varphi \int_{-\infty}^{\infty} \mathrm{d} \xi \int_{-1}^{1} \frac{\left(1-\underline{z}^{2}\right)}{(\cosh \xi-\underline{z})^{2}} \mathrm{~d} \underline{z}
$$

Making firstly the integration over $\underline{z}$ and $\xi$ in the finite limits $[-\bar{\xi}, \bar{\xi}]$ we obtain

$$
\int_{-\infty}^{\infty} \mathrm{d} \xi \int_{-1}^{1} \frac{\left(1-\underline{z}^{2}\right)}{(\cosh \xi-\underline{z})^{2}} \mathrm{~d} \underline{z}=\lim _{\bar{\xi} \rightarrow \infty}\left[4\left(\ln \frac{\cosh \bar{\xi}+1}{\cosh \bar{\xi}-1}\right) \sinh \bar{\xi}\right]=8 .
$$

Thus we have

$$
M_{z}=2 b d
$$

As we see, the full angular momentum is independent of the interdyonic distance and their speed! It is evident that this result is typical for any electrodynamics model, i.e. the form of the function $\mathcal{H}(\mathbf{D}, \mathbf{B})$ is immaterial.

The property, that the full angular momentum is independent of the internal movement parameters, permits a consideration of the bidyon as an electromagnetic model for charged particle with spin. The charge of this particle is $2 d$ and its spin is equal to $\left|M_{z}\right|$. We set $2 \bar{d}=e$, were $e$ is the absolute value of the electron charge, and $\left|M_{z}\right|=\frac{\hbar}{2}$. Thus we have

$$
\bar{b} e=\frac{\hbar}{2} \quad \Longrightarrow \quad \bar{b}=\frac{e}{2} \frac{\hbar}{e^{2}}=\frac{e}{2} \frac{1}{\bar{\alpha}} \Longrightarrow \frac{\bar{d}}{\bar{b}}=\bar{\alpha}
$$

where $\bar{\alpha}=e^{2} / \hbar \approx 0.00729735$ is the fine structure constant.

Now we shall find the trajectory $a\left(x^{0}\right)$ in initial approximation. For this purpose we use the integral energy conservation law for full three-dimensional space. The full energy of initial field configuration (9.1) is depend on the distance between the dyons $a$ and on the speed $V: \mathcal{E}=\mathcal{E}(a, V)$. On the trajectory $a\left(x^{0}\right)$ the full energy is constant. Thus we can obtain the trajectory with help the following formula:

$$
x^{0}=\int_{a(0)}^{a} \frac{\mathrm{d} \tilde{a}}{V(\tilde{a}, \mathcal{E})}
$$

where $V(a, \mathcal{E})$ is the inverse function to the function $\mathcal{E}(a, V)$. 
It is evident that we have a family of the trajectories for all possible values of the energy.

But in fact, the energy depend on square of the speed. Thus we can obtain the acceleration from the function $\mathcal{E}\left(a, V^{2}\right)$ by the following way:

$$
\frac{\mathrm{d} \mathcal{E}}{\mathrm{d} x^{0}}=\frac{\partial \mathcal{E}}{\partial a} V+\frac{\partial \mathcal{E}}{\partial V^{2}} 2 \frac{\mathrm{d} V}{\mathrm{~d} x^{0}} V=0 \quad \Longrightarrow \quad \frac{\mathrm{d} V}{\mathrm{~d} x^{0}}=-\frac{1}{2} \frac{\partial \mathcal{E}}{\partial a} / \frac{\partial \mathcal{E}}{\partial V^{2}} .
$$

Let us obtain the function $\mathcal{E}\left(a, V^{2}\right)$. We shall use a numerical calculation for the energy integral. But at first we obtain some interesting approximate formula for the energy in the case of very small distance between the dyons and their speed is not very close to the speed of light. We substitute the field $\mathcal{D}^{(0)}$ into expression for $\mathcal{H}$ (2.7) and use formulas for bispherical coordinates (9.6) and (9.8). We obtain that as $a^{\prime} \rightarrow 0$ the main term of the radicand is $\alpha^{4}\left(\mathcal{P}_{\varphi}\right)^{2}$, which is proportional to $\left(a^{\prime}\right)^{-8}$. Thus for very small values of $a^{\prime}$ we have the following formula:

$$
\mathcal{E}=\frac{1}{4 \pi} \int\left|\mathcal{P}_{\varphi}\right|(\rho \mathrm{d} z \mathrm{~d} \rho \mathrm{d} \varphi)=\frac{\bar{b} \bar{d}}{8 \pi a^{\prime}} \int_{-\pi}^{\pi} \mathrm{d} \varphi \int_{-\infty}^{\infty} \mathrm{d} \xi \int_{-1}^{1} \frac{\sqrt{1-\underline{z}^{2}}}{(\cosh \xi-\underline{z})} \mathrm{d} \underline{z}
$$

Making firstly the integration over $\xi$ we obtain $\int_{-\infty}^{\infty} \mathrm{d} \xi \int_{-1}^{1} \frac{\sqrt{1-\underline{z}^{2}}}{(\cosh \xi-\underline{z})} \mathrm{d} \underline{z}=$

$$
=2 \int_{-1}^{1}\left[\arctan \left(\sqrt{\frac{1+\underline{z}}{1-\underline{z}}}\right)-\arctan \left(-\sqrt{\frac{1+\underline{z}}{1-\underline{z}}}\right)\right] \mathrm{d} \underline{z}=2 \pi .
$$

As result we have the following formula:

$$
\mathcal{E}=\frac{\pi \bar{b} \bar{d} \sqrt{1-V^{2}}}{2 a}
$$

As we see, in the case of $V \neq 1$ this formula gives that $\mathcal{E} \rightarrow \infty$ as $a \rightarrow 0$ ! Moreover the effective mass of this system is negative, in the sense that the acceleration (see (9.15)) is directed to the region with a greater rest energy!

Notice, the sum $\stackrel{1}{\mathcal{D}}+\stackrel{2}{\mathcal{D}}$ are considered here as initial approximation, in particular, for very small distance between the singularities $(2 a \ll \bar{r})$. However, as indicated above (see the end of section 7) this may be rightful, because absolute value of the dyon's fields D, B are infinity at the singular point. Thus we can hope that our iterative procedures (7.4) or (7.7) with this initial approximation can give an approximated solution. In any case the investigation of field configuration (9.1) may give leading considerations for obtaining the solution.

Now we present the results of numerical calculations ${ }^{2}$ for the function $\mathcal{E}\left(a, V^{2}\right)$. We used the dimensional system in which $\alpha=1$ and $\bar{r}=1$. In this dimensional

\footnotetext{
${ }^{2}$ Here we had used FORTRAN program with NAG library (function D01DAF for integration in two dimensions).
} 
system the energy of $\mathcal{D}^{(0)}$ for $a=\infty$ and $V=0$ is $2 \overline{\mathcal{E}}=4 \beta / 3 \approx 2.4721$ (see (4.5)), and equality $a=1$ implies that $a=\bar{r}$ in other dimensional system. According to (9.13), (4.2) we have $d=\bar{\alpha} b, b=\left(1+\bar{\alpha}^{2}\right)^{-\frac{1}{2}}$ in this dimensional system. We made the calculation for $a=0.00004 \div 4$ and $V^{2}=0 \div 0.95$. In order to make the best convergence of the integral (both near the singular points and as $\stackrel{1}{r}, \stackrel{2}{r} \rightarrow \infty$ ), we divide the space of integration on two part. In the first part, which is bounded by the sphere with radius $20 \div 40$, we used the bispherical coordinates. And outside this sphere but inside the sphere with radius $200 \div 1000$ we used the spherical coordinates. The precision for obtained results of calculations is no more than $5 \%$.

As noted above the effective mass for the internal movement of the dyons in $\mathcal{D}^{(0)}$ may be negative for very small distance between the dyons. But, of course, as $a \rightarrow \infty$ this mass is positive. Thus there are an inflectional line in coordinates $\left(a, V^{2}\right)$ on which the mass is equal to zero. We denote the point of intersection this line with the axes $a$ as $\bar{a}$. That is

$$
\left.\frac{\partial \mathcal{E}\left(a, V^{2}\right)}{\partial V^{2}}\right|_{\begin{array}{c}
a \\
= \\
V
\end{array}=0}=0 \quad .
$$

According our numerical calculations $\bar{a} \approx 4.6 \cdot 10^{-3}$ and value of the energy $\mathcal{E}(\bar{a}, 0)$ is near $4 \beta / 3$. It is possible that this is her exact value. This point $(\bar{a}, 0)$ in some sense is dual to the point $(\infty, 0)$ where the energy is $\mathcal{E}=2 \overline{\mathcal{E}}$. We shall also see this duality below in the context of obtained trajectories $a\left(x^{0}\right)$.

The numerical calculations give that for $a<\bar{a}$ and $V=0$ the values of the energy practically equal to ones given by formula (9.18). But this agreement disappears as the speed $V$ increase. The appropriate results show in figure 3 . (The values calculated by formula (9.18) show with discontinuous line.)
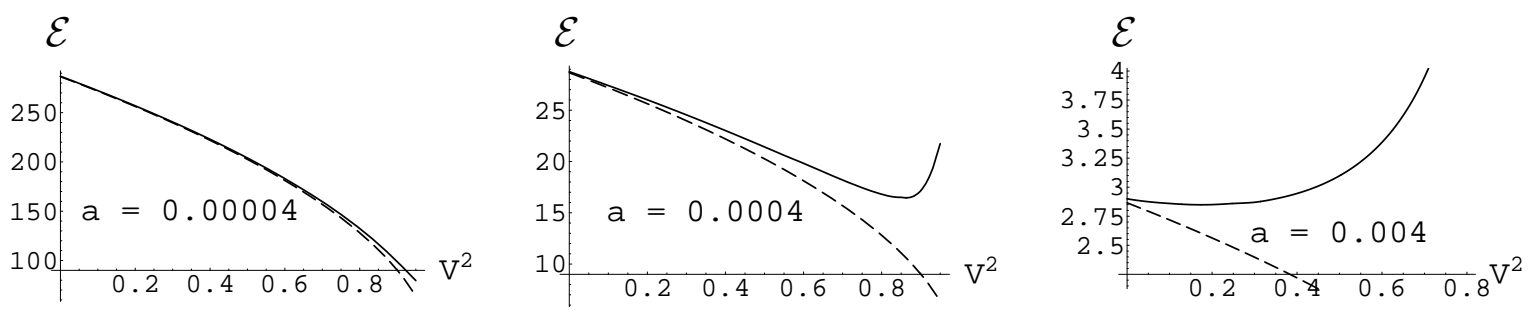

Figure 3: Energy of $\mathcal{D}^{(0)}$ for $a<\bar{a}$ (approximation by formula (9.18)).

The best agreement with the numerical results is given by addition the term $C \frac{V^{2}}{1-V^{2}}$ to expression (9.18) for the energy. Here the coefficient $C$ is chosen from the condition $\mathcal{E}(\bar{a}, 0)=2 \overline{\mathcal{E}}$. Thus we have the following approximate formula for the energy:

$$
\mathcal{E}=\frac{\pi \bar{b} \bar{d} \sqrt{1-V^{2}}}{2 a}+\frac{2}{3} \beta \alpha^{-2} \bar{r}^{3} \frac{V^{2}}{1-V^{2}}
$$


According this formula we have the following expression:

$$
\bar{a}=\frac{3 \alpha^{2} \pi \bar{b} \bar{d}}{8 \beta \bar{r}^{3}}
$$

In our dimensional system this formula gives $\bar{a}=4.63673 \cdot 10^{-3}$ that good agree with the numerical result. By addition the terms $\sim\left(\frac{V^{2}}{1-V^{2}}\right)^{n}$, where $n=2,3,4$, to formula $(9.20)$ we can obtain a formula, which gives very good approximation for the results of numerical calculations (for $a<\bar{a}$ and $V^{2}<0.95$ ). This formula has the following form:

$$
\mathcal{E}=\frac{0.0115 \sqrt{1-V^{2}}}{a}+\frac{1.236 V^{2}}{1-V^{2}}-\frac{0.083 V^{4}}{\left(1-V^{2}\right)^{2}}+\frac{0.007 V^{6}}{\left(1-V^{2}\right)^{3}}-\frac{0.0002 V^{8}}{\left(1-V^{2}\right)^{4}}
$$

Here we change the two factors in formula (9.20) for their numerical values in our dimensional system. In figure 4 is shown this good agreement between the results of numerical calculations and values calculated by formula (9.22).
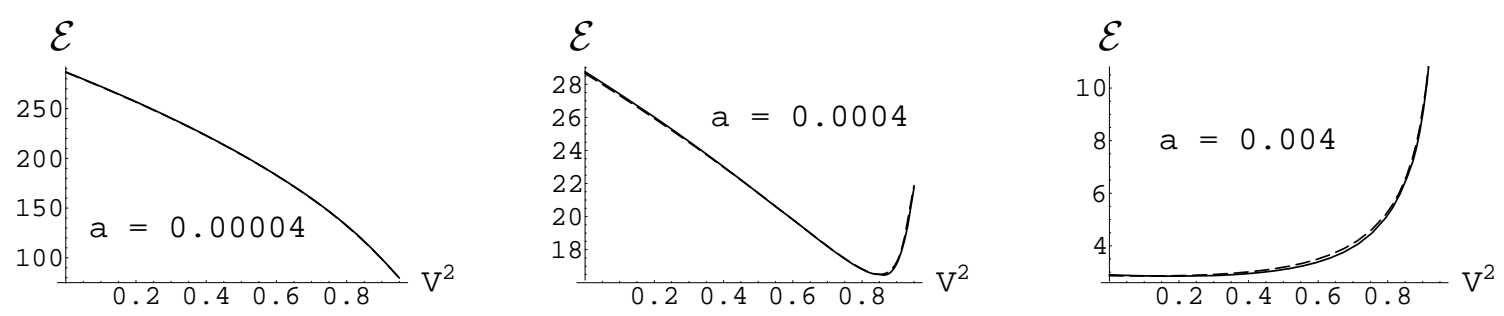

Figure 4: Energy of $\mathcal{D}^{(0)}$ for $a<\bar{a}$ (approximation by formula (9.22)).

It is evident that formula (9.22) gives the same expression for $\bar{a}$ (9.21).

In the region of $a>\bar{a}$ the function $\mathcal{E}\left(a, V^{2}\right)$ has a minimum. The appropriate plot for $V=0$ is shown in figure 5 .

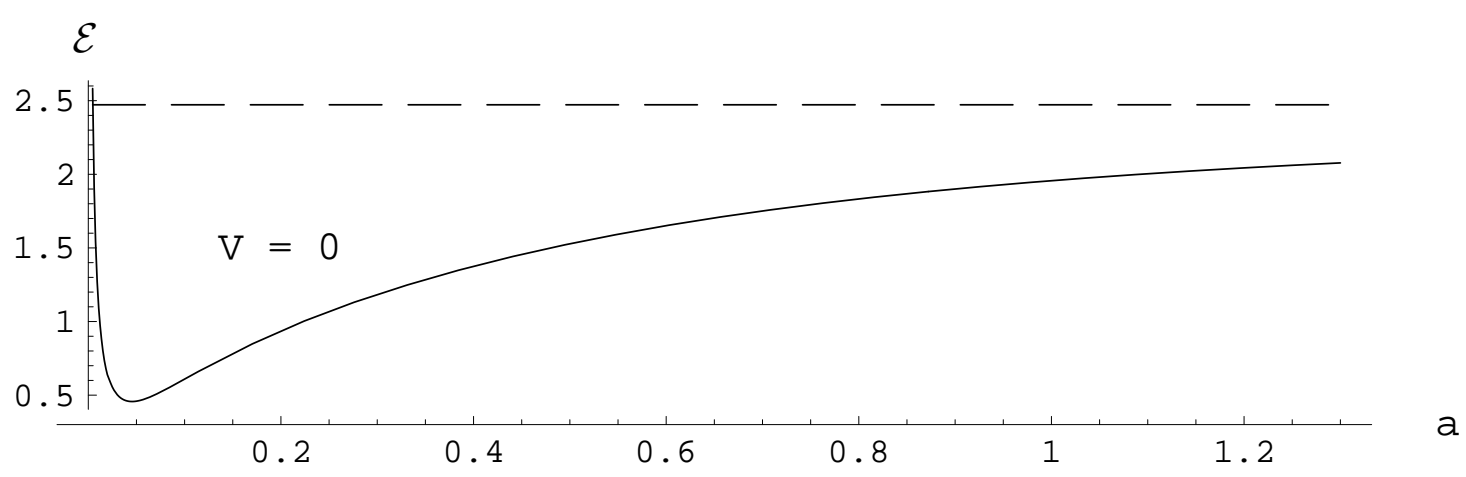

Figure 5: Energy of $\mathcal{D}^{(0)}$ for middle values of $a$ and $V=0$.

As we see, the energy falls very quick and then it increases very slow as the values of $a$ increase. Such dependence is convenient to show by the plot with logarithmic 
scale on $a$. In figure 6 is shown the function $\mathcal{E}\left(\log _{10} a, V^{2}\right)$ for $V^{2}=0 \div 0.6$ with step 0.05 . Here we have the minimal value of the energy $\mathcal{E}_{m} \approx 0.46$ for

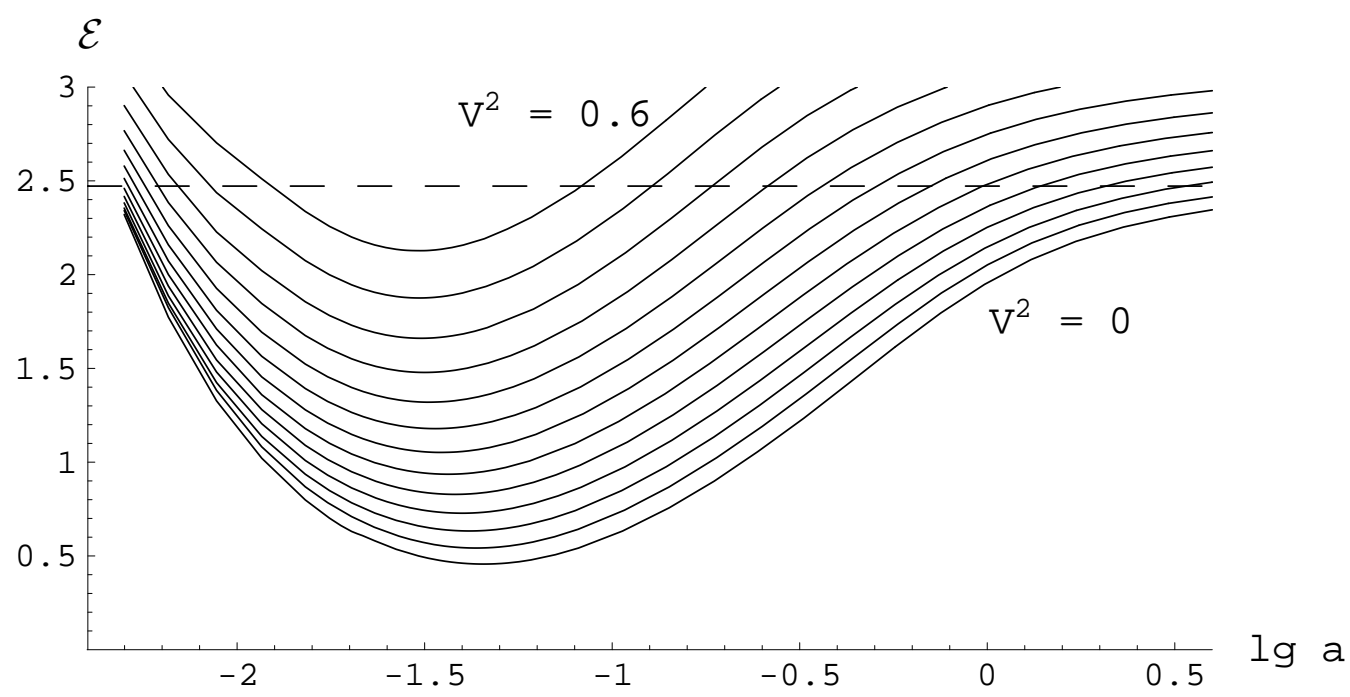

Figure 6: The energy in logarithmic scale of $a$ and $V^{2}=0 \div 0.6$ with step 0.05 .

$a=a_{m} \approx 0.045$. For very small value of deviation $\left|a-a_{m}\right|$ we have the following approximate formula:

$$
\mathcal{E}=\mathcal{E}_{m}+128\left(a-a_{m}\right)^{2}+1.65 V^{2}
$$

Now we investigate the trajectories for various values of the energy. With the numerical calculations we have obtained the function $V(a, \mathcal{E})$ for the region of $a$ which is shown in figure 6. The appropriate results are shown in figure 0. As we

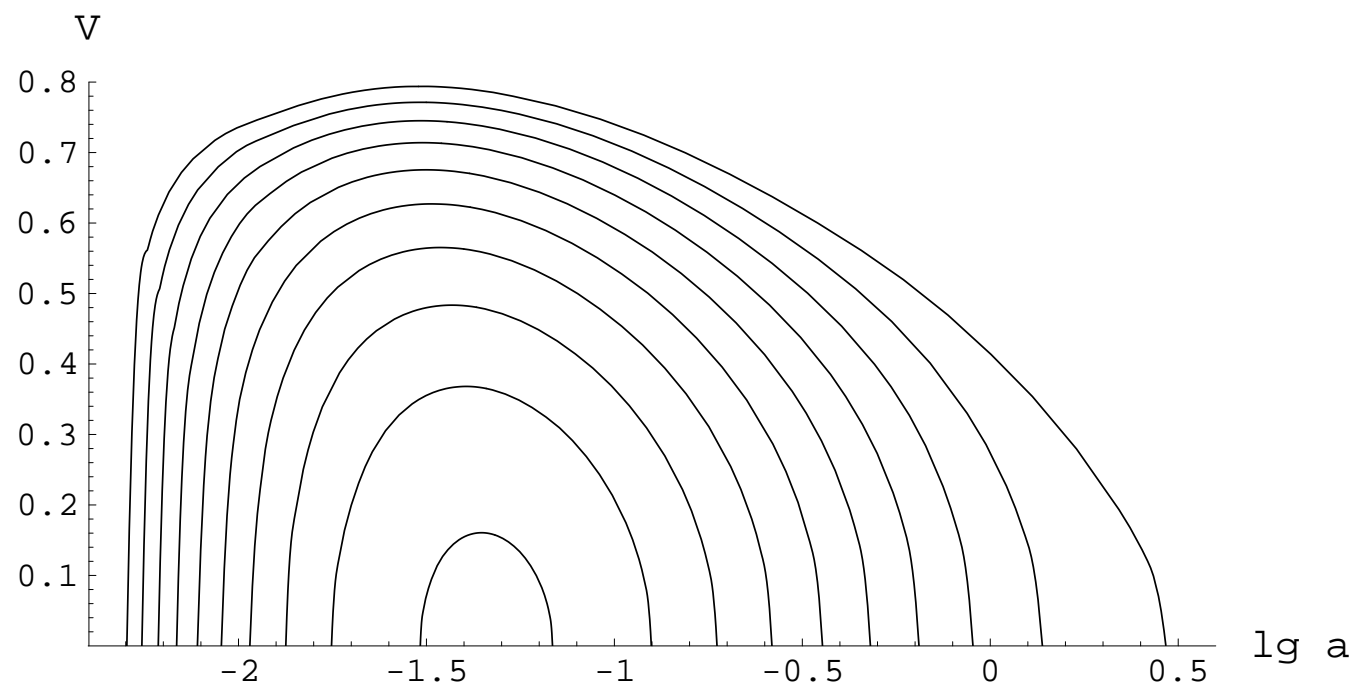

Figure 7: The function $V\left(\log _{10} a, \mathcal{E}\right)$ for $\mathcal{E}=0.5 \div 2.3$ with step 0.2 . 
see, here we have a periodical motion. According to formula (9.14) the period of this motion is given by the formula

$$
T=2 \int_{a_{\min }}^{a_{\max }} \frac{\mathrm{d} \tilde{a}}{V(\tilde{a}, \mathcal{E})} \quad \text { and } \quad \omega \equiv \frac{2 \pi}{T} .
$$

For the minimal value of the energy $\mathcal{E}_{m}$ we calculate the circular frequency $\omega$ using formula (9.23). The appropriate value is 8.825. And for values of the energy from 0.5 to 2.3 with step 0.1 we numerically calculate the frequency with help of formula $(9.24)^{3}$. As result we obtain the function $\omega(\mathcal{E})$. The obtained dependence $\omega(\mathcal{E})$ is shown in figure 8 . The dual points $(a, V)=(\bar{a}, 0)$ and $(\infty, 0)$ are unstable points of rest with the energy $2 \overline{\mathcal{E}}$. The appropriate period is infinity and the frequency is zero.

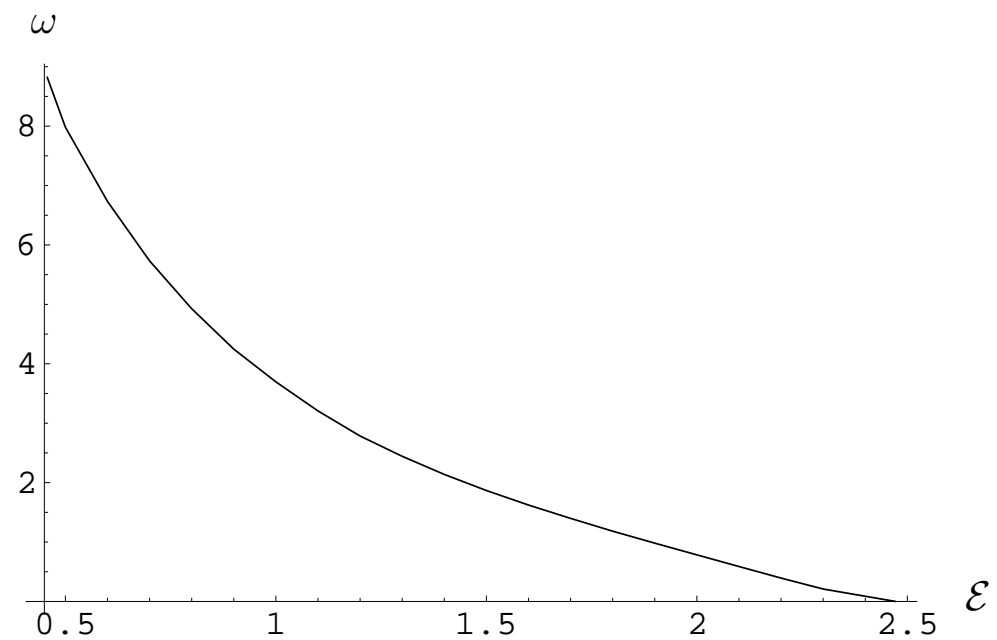

Figure 8: The function $\omega(\mathcal{E})$ of the periodical motion for $\mathcal{E}<4 \beta / 3$.

Now we consider the movement in the region $a<\bar{a}$. The lines with constant energy for this region are shown in figure 9. Let us consider the line in figure 10 that correspond to sole value of the energy. As we see in this figure, if for an initial instant of time the speed is zero $(V=0)$, then the dyons begin to approach each other from the points $a=a_{\iota}(\mathcal{E})$ and quick gather a high speed. According to the all formulas for the energy in the region $a<\bar{a}$ ( (9.18), (9.20) and (9.22) ) we have the following expression:

$$
a_{\prime}=\frac{\pi \bar{b} \bar{d}}{2 \mathcal{E}}
$$

But at any intermediate point between $a_{\prime \prime}$ and $a_{\prime}$ in figure 10 the dyons may have two different values of the speed for one value of the energy. In principle, the

\footnotetext{
${ }^{3}$ In fact we numerically calculated the integral from $a_{\min }+\delta a_{1}$ to $a_{\max }-\delta a_{2}$. For the regions $\left[a_{\min }, a_{\min }+\delta a_{1}\right],\left[a_{\max }-\delta a_{2}, a_{\max }\right]$ we approximated the function $\mathcal{E}\left(a, V^{2}\right)$ by a linear dependence on $a, V^{2}$ and analytically calculate the appropriate integral.
} 


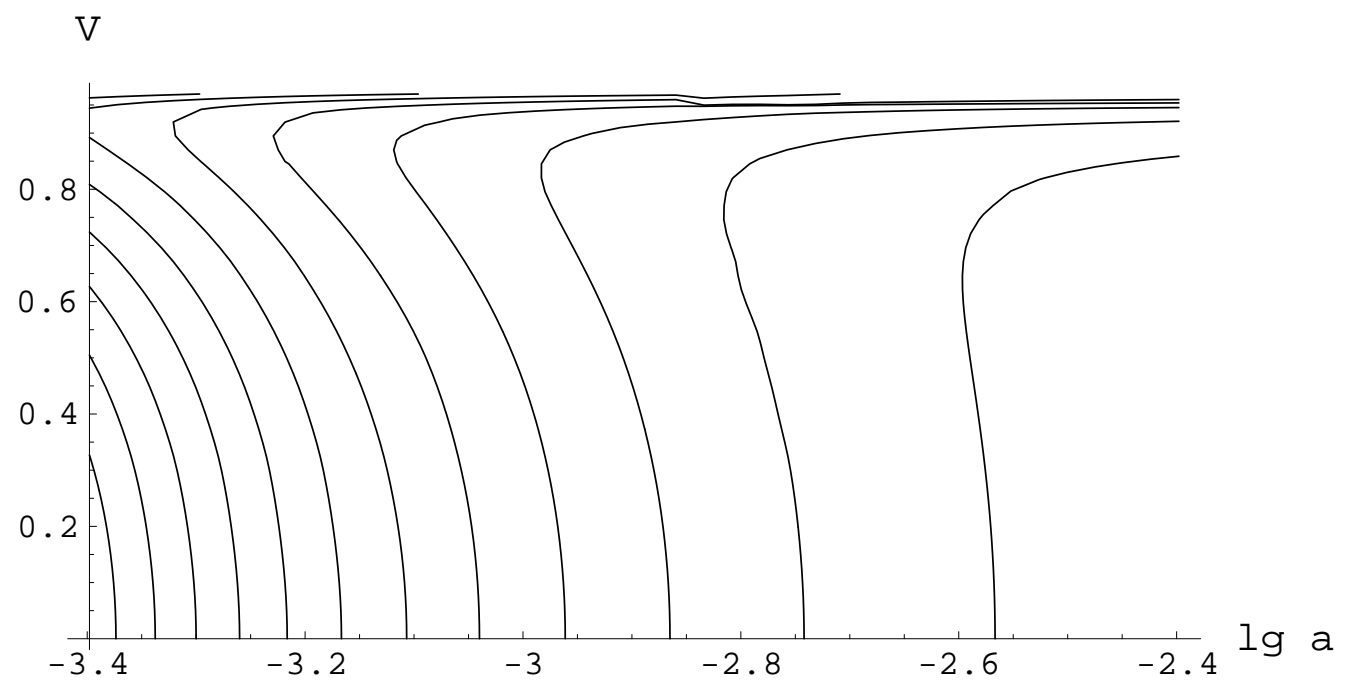

Figure 9: Lines with constant energy for $a<\bar{a}$.

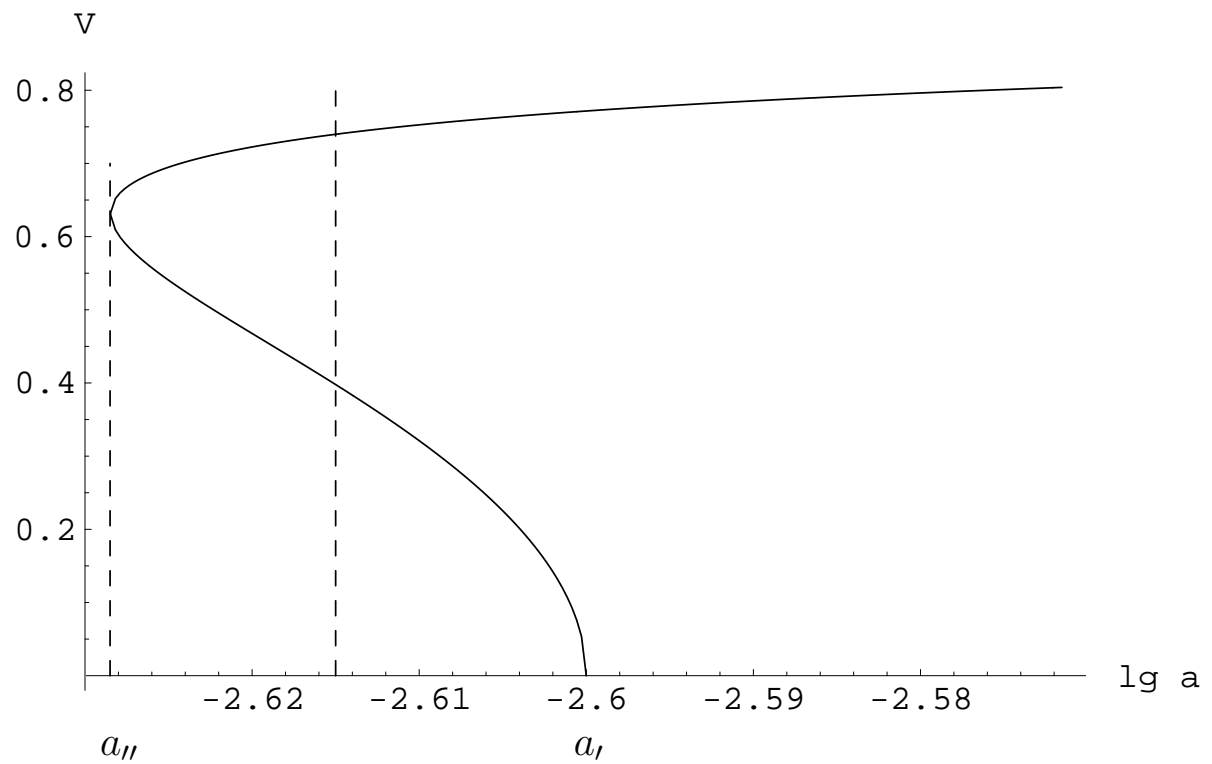

Figure 10: Line with constant energy for $a<\bar{a}$.

system may jump from one branch of the constant energy line to another. And in the initial approximation there are not any criterion for choosing between these branches. But in first approximation we take into account a radiation of the dyons. Because an amplitude of the radiation depend on acceleration, we can propose that this radiation will prevent for the jumping (with infinite acceleration). Thus we believe that the bidyon system in initial approximation moves from the point $a=a_{\iota}, V=0$ to the point $a_{\prime \prime}$ on the bottom (in the figure) branch of the constant energy line. At the point $a=a_{\| \prime}$ the acceleration on the trajectory is infinity and direction of the 
velocity is changed to opposite one. From the point $a_{\prime \prime}$ the dyons may move on the top branch $(a \rightarrow \infty)$ or come back on the bottom one. Of course, near this point the radiation plays a significance role for the movement. Thus we can suppose that the bidyon dynamical system stop short of the point with infinite acceleration and come back on the branch with less speed, that is the bottom branch. In this case we have the periodical motion between the points $a_{l}$ and $a_{l \prime}$.

We shall numerically calculate the period for this periodical motion. But at first we shall have obtained some analytical result for an idealized case. If we have a dynamical system with energy given by formula (9.18), then we have her trajectory with the formula

$$
x^{0}=\int_{a_{1}}^{a}\left[1-\left(\frac{2 \tilde{a} \mathcal{E}}{\pi \bar{b} \bar{d}}\right)^{2}\right]^{-\frac{1}{2}} \mathrm{~d} \tilde{a}=\left.\frac{\pi \bar{b} \bar{d}}{2 \mathcal{E}} \arcsin \left(\frac{2 \tilde{a} \mathcal{E}}{\pi \bar{b} \bar{d}}\right)\right|_{a_{\prime}} ^{a}
$$

Using formula (9.25) for $a$, we can obtain the following trajectory:

$$
a=\frac{2}{\omega}\left|\cos \frac{\omega}{2} x^{0}\right| \quad, \quad \omega=\frac{4 \mathcal{E}}{\pi \bar{b} \bar{d}}=\frac{16 \mathcal{E}}{\pi \hbar}
$$

As we see, in this case the energy is proportional to the frequency! The system moves from the point $a=a$, to the point $a=0$ and come back. In keeping with the conservation of full angular momentum, the dyons may not change places. At the point $a=0$ the speed is equal to the speed of light $V=1$, for this idealized system.

Now we present a dependence $\omega(\mathcal{E})$ that numerically calculated by formula (9.22) for the energy. As noted, this formula very good approximate the numerically calculated function $\mathcal{E}(a, V)$. In figure 11 we have the plot for numerically calculated function $\omega(\mathcal{E})$ (continuous line), asymptote $\mathcal{E}=2 \overline{\mathcal{E}}$, and dependence $\omega(\mathcal{E})$ for idealized motion (9.27) (discontinuous lines).

As we see, $\omega \rightarrow \infty(T \rightarrow 0)$ when the energy approached $2 \overline{\mathcal{E}}$ from above, in contrast to the case when the energy approached $2 \overline{\mathcal{E}}$ from below.

We can derive also a formula that will better approximate for the numerically calculated function $\omega(\mathcal{E})$. Let us take movement (9.26) of idealized system (9.18). But we shall suppose that the system stop short of the point $a=0$ and come back from some point $a_{\| \prime} \neq 0$. We shall have obtained this point from approximate formula (9.20). This formula considered as equation with their associated equation $\frac{\partial \mathcal{E}}{\partial V^{2}}=0$ are the system for obtaining of the point $\left(a_{\prime \prime}, V_{\|}^{2}\right)$ with infinity acceleration. The appropriate solution is

$$
a_{\prime \prime}=\frac{3 \sqrt{3} \pi \bar{b} \bar{d} \sqrt{\overline{\mathcal{E}}}}{4(\mathcal{E}+\overline{\mathcal{E}})^{\frac{3}{2}}} \quad, \quad V_{\prime \prime}^{2}=\frac{\mathcal{E}-2 \overline{\mathcal{E}}}{\mathcal{E}+\overline{\mathcal{E}}}
$$




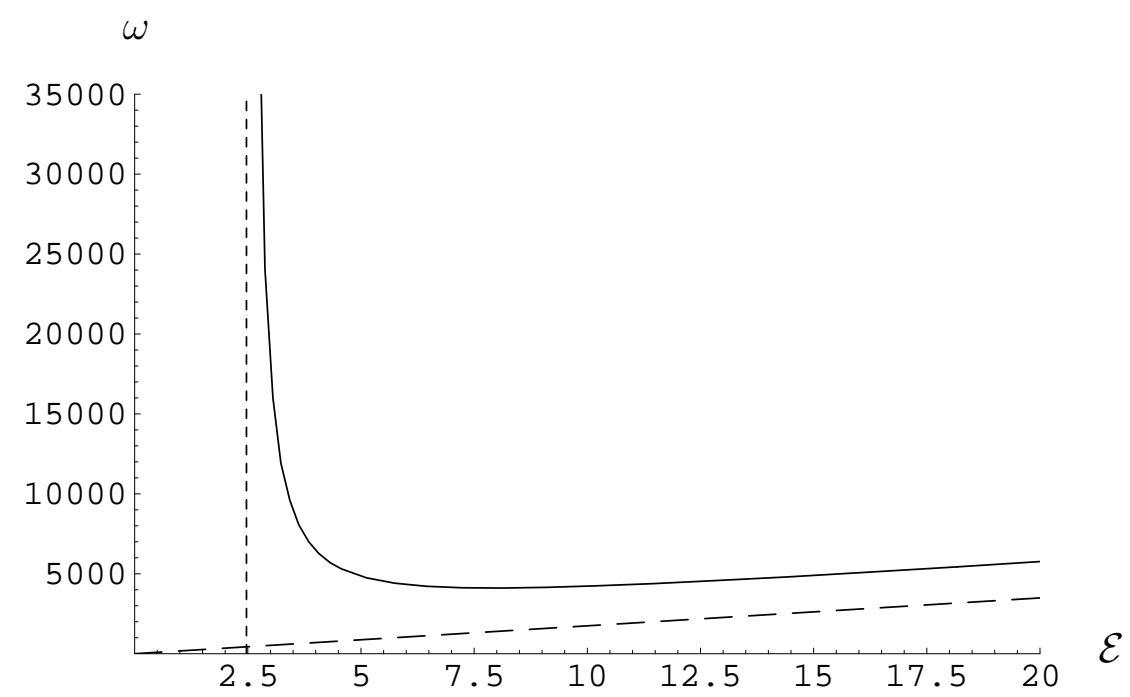

Figure 11: Function $\omega(\mathcal{E})$ for the periodical motion in $a<\bar{a}$.

Using expression (9.26) we have the period for this restricted idealized motion in the following form:

$$
T=\frac{\pi \bar{b} \bar{d}}{\mathcal{E}}\left[\frac{\pi}{2}-\arcsin \left(\frac{2 a_{\prime \prime} \mathcal{E}}{\pi \bar{b} \bar{d}}\right)\right]
$$

And for the circular frequency we have

$$
\omega=\frac{2 \mathcal{E}}{\bar{b} \bar{d}} /\left\{\frac{\pi}{2}-\arcsin \left[\frac{3 \sqrt{3 \overline{\mathcal{E}} \mathcal{E}}}{2(\mathcal{E}+\overline{\mathcal{E}})^{\frac{3}{2}}}\right]\right\} .
$$

In figure 12 is shown this function $\omega(\mathcal{E})$ (discontinuous curve) with the numerically calculated function according to formula (9.22) for the energy (continuous curve). As we see, there are some distinction between the plots near $\mathcal{E}=2 \overline{\mathcal{E}}$. But for superior value of the energy we have good agreement between the plots. For high values of the energy formula $(9.30)$ gives

$$
\omega \approx \frac{4 \mathcal{E}}{\pi \bar{b} \bar{d}}+\frac{12 \sqrt{3 \overline{\mathcal{E}}}}{\pi^{2} \bar{b} \bar{d}} \sqrt{\mathcal{E}}
$$

Thus we have considered the internal movement of the bidyon for initial approximation, which is the sum of the dyon's fields $\stackrel{1}{\mathcal{D}}+\stackrel{2}{\mathcal{D}}$. The appropriate first approximation is connected with the radiation of the dyons. The oscillating bidyon in initial approximation may give rise to some standing wave in first approximation. According to properties of the radiation for accelerated charge, an amplitude of this standing wave is zero at infinity of three-dimensional space. In this sense we can call it as restricted standing wave. The standing wave may change the trajectory $a\left(x^{0}\right)$. 


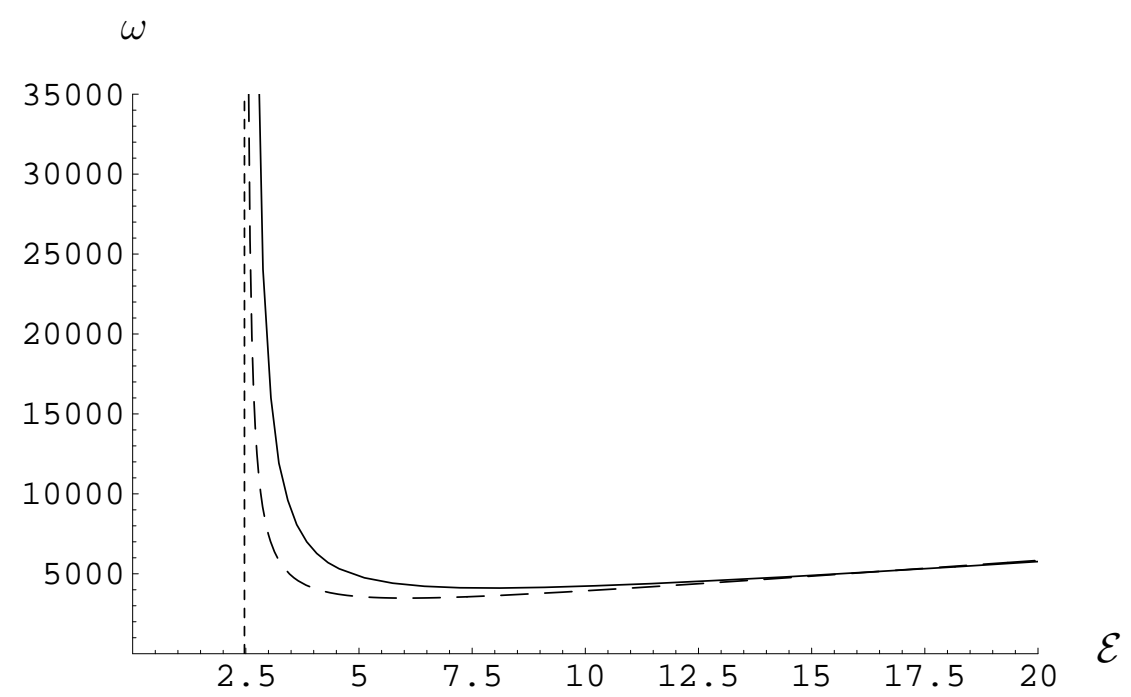

Figure 12: The function $\omega(\mathcal{E})$ for $a<\bar{a}$ with its approximation.

Thus we shall have an energy interchange between the initial approximation and the standing wave. Some equilibrium oscillating field configuration may exist for this case. Because this problem has two-point boundary conditions, it is possible that the bidyon oscillating system will have some discrete set of allowable frequencies.

Furthermore, the oscillating bidyon will interact with any plane waves, which are exact solutions of the model [3]. The resonance interaction will intercept the plane waves with bidyon's frequencies. As result we shall have some non-restricted (in three-dimensional space) standing wave connected with the oscillating bidyon. Thus it is valid to say that such oscillating field configuration occupies whole threedimensional space. Of course, near the singular points this configuration has a localization region, but the associated non-restricted standing wave has some finite amplitude at infinity.

We can represent the oscillating bidyon solution by Fourier series in time coordinate. Thus we have the following series for the solution in own coordinate system $y^{\mu}$ :

$$
\underline{\mathcal{D}}=\sum_{f=-\infty}^{\infty} \underline{\mathcal{D}}_{f} \exp \left(-\mathrm{i} f \underline{\omega} y^{0}\right)
$$

where $\underline{\omega} \equiv \frac{2 \pi}{T}, \underline{\mathcal{D}}_{f}=\underline{\mathcal{D}}_{f}\left(y^{i}, \underline{\omega}\right)$, are complex column-function given by the formula

$$
\underline{\mathcal{D}}_{f}=\frac{1}{2 \pi} \int_{0}^{T} \underline{\mathcal{D}} \exp \left(\mathrm{i} f \underline{\omega} y^{0}\right) \mathrm{d} y^{0}
$$

With help Lorentz transformation we can obtain from (9.32) the following moving 
bidyon solution:

$$
\mathcal{D}=\sum_{f=-\infty}^{\infty} \mathcal{D}_{f} \exp (\mathrm{i} f \Theta)
$$

where $\Theta=k_{\mu} x^{\mu}, k_{\mu} k^{\mu}=-\underline{\omega}^{2}$, and the fields $\mathbf{D}_{f}, \mathbf{B}_{f}$ (for the column $\mathcal{D}_{f}$ ) are connected with the fields $\underline{\mathbf{D}}_{f}, \underline{\mathbf{B}}_{f}$ (for the column $\underline{\mathcal{D}}_{f}$ ) by formulas of Lorentz transformation (4.6a) with functions $\mathbf{E}(\mathbf{D}, \mathbf{B}), \mathbf{H}(\mathbf{D}, \mathbf{B})$ (2.6).

\section{Effect of gravitational interaction}

In the article [3] we had considered the distortion of a small amplitude quickoscillating wave against the background of a high field, for this nonlinear electrodynamic model. We had shown that this distortion coincide with light beams distortion in gravitational field with the following metric:

$$
\bar{g}^{\mu \nu}=g^{\mu \nu}-\alpha^{2} T^{\mu \nu}
$$

where $g^{\mu \nu}$ is the metric of basic Minkowskian space and the energy-momentum tensor $T^{\mu \nu}$ is formulated from the background field.

Bidyon solution (9.34) has the quick-oscillating wave part. As distinct from light wave, this wave has the rest frequency $\underline{\omega}$. But we can presume that this wave (in the region with small amplitude of it) will have the analogous behavior that light wave.

Thus let us consider the problem for propagation of the small amplitude quickoscillating wave with the rest frequency against a high field background. Here we shall use a method that some distinct from one, which used in the article [3]. Let us take the cartesian coordinate system $\left\{x^{\mu}\right\}$ with the metric $g^{\mu \nu}=h^{\mu \nu}$ such that

$$
\left|h_{00}\right|=\left|h_{11}\right|=\left|h_{22}\right|=\left|h_{33}\right|=1 \quad, \quad h_{\mu \nu}=0 \quad \text { for } \quad \mu \neq \nu .
$$

In this section we don't define one of two signatures of the Minkowskian metric: $(-+++)$ or $(+---)$.

In this coordinate system we have the following form of equation (2.12) for the potential $A_{\mu}$ outside of the singular set:

$$
C^{\mu \nu \sigma \rho} \frac{\partial^{2} A_{\rho}}{\partial x^{\mu} \partial x^{\sigma}}=0 .
$$

Let we have a small field configuration $\underline{\tilde{A}}_{\mu}\left(y^{\nu}\right)$, which is periodical on $y^{0}$ with circular frequency $\underline{\omega}$ and satisfies the Maxwell equations

$$
\bar{C}^{\mu \nu \sigma \rho} \frac{\partial^{2} \underline{\tilde{A}}_{\rho}}{\partial y^{\mu} \partial y^{\sigma}}=0
$$


where

$$
\bar{C}^{\mu \nu \sigma \rho}=h^{\mu \sigma} h^{\nu \rho}-h^{\mu \rho} h^{\nu \sigma}
$$

This field configuration may represent the quick-oscillating part of the bidyon solution (9.32) in the region where the electromagnetic field is small and the nonlinearity can be neglected. With help the Lorentz transformation we can obtain also an appropriate moving field configuration. But if we have its moving against the background of a high field, we can presume a change for parameters of this small field configuration. Let us take a solution of the model as the background field $\stackrel{\circ}{A}_{\mu}$ and a moving field configuration $\tilde{A}_{\mu}\left(x^{\nu}\right)$, which is related with $\underline{\tilde{A}}_{\mu}\left(y^{\nu}\right)$ by some unknown transformation. We substitute their sum

$$
A_{\mu}=\stackrel{\circ}{A}_{\mu}+\tilde{A}_{\mu}
$$

into system of equation (10.3). Taking into account that for the associated electromagnetic field $\left|\tilde{F}_{\mu \nu}\right| \ll\left|\stackrel{\circ}{F}_{\mu \nu}\right|$ and $\left|\frac{\partial \tilde{F}_{\mu \nu}}{\partial x^{\delta}}\right|\left|\stackrel{\circ}{F}_{\sigma \rho}\right| \gg\left|\frac{\partial \stackrel{\circ}{F}_{\mu \nu}}{\partial x^{\delta}}\right|\left|\tilde{F}_{\sigma \rho}\right|$ we obtain the following equation:

$$
C^{\mu \nu \sigma \rho} \frac{\partial^{2} \tilde{A}_{\rho}}{\partial x^{\mu} \partial x^{\sigma}}=0
$$

where coefficients $C^{\mu \nu \sigma \rho}(2.13)$ include the background field $\stackrel{\circ}{F}_{\mu \nu}$ only.

Into some restricted region, where we can believe that the background field is constant, we can try to find a coordinate transformation

$$
\mathrm{d} y^{\mu}=G_{. \nu}^{\mu} \mathrm{d} x^{\nu} \quad, \quad \mathrm{d} x^{\mu}=\check{G}_{. \nu}^{\mu} \mathrm{d} y^{\nu},
$$

which transform system of equations (10.6) to system (10.4). We shall have this transformation if

$$
C^{\mu \nu \sigma \rho}=\check{G}_{. \delta}^{\mu} \check{G}_{. \gamma}^{\nu} \check{G}_{. \zeta}^{\sigma} \check{G}_{. \xi}^{\rho} \bar{C}^{\delta \gamma \zeta \xi}=\bar{g}^{\prime \mu \rho} \bar{g}^{\prime \nu \sigma}-\bar{g}^{\prime \mu \sigma} \bar{g}^{\prime \nu \rho}
$$

where $\bar{g}^{\prime \mu \rho} \equiv \check{G}_{. \zeta}^{\mu} \check{G}_{. \xi}^{\rho} h^{\zeta \xi}$ is a metric associated with this transformation.

With help the direct substitution we obtain that $\bar{g}^{\prime \mu \rho}=\bar{g}^{\mu \rho}$, where the metric $\bar{g}^{\mu \rho}$ is defined by formula (10.1)!

Let us write the basic relations, which connect the metric $\bar{g}^{\mu \rho}$ with the transformation matrixes.

$$
\bar{g}^{\mu \nu} G_{. \mu}^{\sigma} G_{. \nu}^{\rho}=h^{\sigma \rho} \quad, \quad \bar{g}^{\mu \rho} \equiv \check{G}_{. \zeta}^{\mu} \check{G}_{. \xi}^{\rho} h^{\zeta \xi}
$$

Thus we have a solution of system (10.6) into the restricted region (where $\bar{g}^{\mu \nu} \approx$ const ) in the following form:

$$
\tilde{A}_{\mu}=G_{. \mu}^{\nu} \underline{\tilde{A}}_{\nu}\left(y^{\sigma}\right)
$$


The metric $\bar{g}^{\mu \nu}$, calculated by formula (10.1) with the background field for full four-dimensional space, define some curvilinear Riemann space, in general case. Thus, according to known theorem of Riemann geometry, we can't consider $G^{\mu \nu}$ as coordinate transformation tensor for full space. But we can always make the transformation to the cartesian coordinates $\left\{y^{\mu}\right\}$ for one point of four-dimensional space. As this point we take the point $\left\{\bar{x}^{\mu}\right\}$, where $\bar{x}^{i}$ are three-dimensional coordinates for some central point in a localization region of the field $\tilde{A}_{\mu}$ at the time moment $\bar{x}^{0}$. Thus for using solution (10.10) in full four-dimensional space we must take $G_{. \mu}^{\nu}=G_{. \mu}^{\nu}\left(\bar{x}^{\rho}\right)$ in formula (10.10). But because $\bar{x}^{i}=\bar{x}^{i}\left(\bar{x}^{0}\right)$ we have $G_{. \mu}^{\nu}=G_{. \mu}^{\nu}\left(\bar{x}^{0}\right)$ into formula (10.10). For the coordinates $\left\{y^{\mu}\right\}$ we take the following expressions:

$$
\begin{array}{rlrl}
y^{i} & =G_{j}^{i}\left(x^{j}-\bar{x}^{j}\right), \quad G_{j}^{i}=G_{j}^{i}\left(\bar{x}^{0}\right), & \bar{x}^{i}=\bar{x}^{i}\left(\bar{x}^{0}\right), \\
y^{0} & =\int \bar{k}_{\mu} \mathrm{d} x^{\mu}, & \bar{k}_{\mu}\left(x^{\nu}\right) & \equiv G_{\mu}^{0}\left(x^{\nu}\right) .
\end{array}
$$

Because the solution $\underline{\tilde{A}}_{\nu}\left(y^{\sigma}\right)$ is periodical by $y^{0}$, the proper time $y^{0}$ play a role of normalized phase for quick-oscillating wave (10.10). In this case the ordinary phase is $\underline{\omega} y^{0}$ and the components of wave vector are $\underline{\omega} \bar{k}_{\mu}$. We have also the following evident relations:

$$
\frac{\partial \bar{k}_{\mu}}{\partial x^{\nu}}=\frac{\partial \bar{k}_{\nu}}{\partial x^{\mu}}
$$

According to formulas (10.9) and definition for $\bar{k}_{\mu}$ (10.11) we have

$$
\bar{g}^{\mu \nu} \bar{k}_{\mu} \bar{k}_{\nu}=h^{00},
$$

where $\bar{g}^{\mu \nu}=\bar{g}^{\mu \nu}\left(x^{\rho}\right), \bar{k}_{\mu}=\bar{k}_{\mu}\left(x^{\rho}\right)$.

This is dispersion relation for the quick-oscillating wave (see also [3]).

Let us adopt the proper time $y^{0}\left(x^{\mu}\right)$ at the point $\bar{x}^{\mu}$ as parameter of motion $s$ for the localization region of the field $\tilde{A}_{\mu}$. That is $s \equiv y^{0}\left(\bar{x}^{\mu}\right)$. Thus we have the following definition for four-velocity:

$$
U^{\mu} \equiv \frac{\mathrm{d} \bar{x}^{\mu}}{\mathrm{d} s}=\check{G}_{.0}^{\mu}\left(\bar{x}^{\nu}\right)
$$

Definitions for $U^{\mu}$ (10.14), $\bar{k}_{\nu}$ (10.11) and formulas (10.9) give also the following relations for the point $\left\{\bar{x}^{\mu}\right\}$ :

$$
\bar{k}_{\mu} U^{\mu}=1 \quad, \quad U^{\mu}=h^{00} \bar{g}^{\mu \nu} \bar{k}_{\nu} .
$$

Let us introduce the inverse tensor $\check{\bar{g}}_{\mu \nu}$ for the tensor $\bar{g}^{\mu \nu}$ :

$$
\check{\bar{g}}_{\mu \nu} \bar{g}^{\nu \rho}=\delta_{\mu}^{\rho} .
$$

Of course, within the limits of the introduced Riemann space the quantities $\check{\bar{g}}_{\mu \nu}$ are components of the metric tensor with inferior indexes. But for the basic Minkowskian 
space the quantities $\bar{g}_{\mu \nu}$ are components of some tensor field and $\check{\bar{g}}_{\mu \nu}$ are components of appropriate inverse one.

From (10.15) and (10.16) we immediately obtain for the point $\left\{\bar{x}^{\mu}\right\}$ that

$$
\check{\bar{g}}_{\mu \nu} U^{\mu} U^{\nu}=h^{00}
$$

Thus we have

$$
\mathrm{d} s^{2}=\frac{1}{h^{00}} \check{\bar{g}}_{\mu \nu} \mathrm{d} \bar{x}^{\mu} \mathrm{d} \bar{x}^{\nu}=\left|\check{\bar{g}}_{\mu \nu} \mathrm{d} \bar{x}^{\mu} \mathrm{d} \bar{x}^{\nu}\right|
$$

From (10.15) we have also

$$
\bar{k}_{\mu}=\frac{1}{h^{00}} \check{\bar{g}}_{\mu \nu} U^{\nu}
$$

Let us obtain an equation for the trajectory $\bar{x}^{\mu}(s)$. Differentiating dispersion relation (10.13) by some coordinate $x^{\rho}$ and using relations (10.12) and expression for $U^{\nu}$ through $\bar{k}_{\mu}$ (10.15) we obtain for the point $\left\{\bar{x}^{\mu}\right\}$

$$
\frac{\partial \bar{g}^{\mu \nu}}{\partial x^{\rho}} \bar{k}_{\mu} \bar{k}_{\nu}+2 h^{00} U^{\nu} \frac{\partial \bar{k}_{\rho}}{\partial x^{\nu}}=0 \quad \Longrightarrow \quad \frac{\partial \bar{g}^{\mu \nu}}{\partial x^{\rho}} \bar{k}_{\mu} \bar{k}_{\nu}+2 h^{00} \frac{\mathrm{d} \bar{k}_{\rho}}{\mathrm{d} s}=0
$$

Substituting (10.19) into (10.20) and using (10.16) we obtain the trajectory equation in the following form:

$$
\frac{\mathrm{d} U^{\mu}}{\mathrm{d} s}+\bar{\Gamma}_{\nu \rho}^{\mu} U^{\nu} U^{\rho}=0
$$

where

$$
\bar{\Gamma}_{\nu \rho}^{\mu}=\frac{1}{2} \bar{g}^{\mu \delta}\left(\frac{\partial \check{\bar{g}}_{\delta \nu}}{\partial x^{\rho}}+\frac{\partial \check{\bar{g}}_{\delta \rho}}{\partial x^{\nu}}-\frac{\partial \check{\bar{g}}_{\nu \rho}}{\partial x^{\delta}}\right) \quad .
$$

As we see, within the limits of the introduced Riemann space (for which $g^{\mu \nu}=\bar{g}^{\mu \nu}$, $\left.g_{\mu \nu}=\check{\bar{g}}_{\mu \nu}, \Gamma_{\nu \rho}^{\mu}=\bar{\Gamma}_{\nu \rho}^{\mu}\right)$ this equation is geodesic line equation!

Notice that here we use the primordial equation for the potential $A_{\mu}$ (10.3) without any gauge condition. It is evident, we can take the gauge condition $\bar{g}^{\mu \nu} \frac{\partial A_{\nu}}{\partial x^{\mu}}=0$ for equation (10.6), which is transformed to the condition $h^{\mu \nu} \frac{\partial \underline{A}_{\nu}}{\partial y^{\mu}}=0$ for equation (10.4).

Thus we have the Riemann space which is induced by background field. The relatively small quick-oscillating wave field configuration with rest frequency and localized near point $\bar{x}^{i}\left(\bar{x}^{0}\right)$ moves on geodesic lines of this Riemann space. This looks as motion of gravitating particles. Thought the bidyon's field is high near the singular points, we may presume that in some cases the quick-oscillating bidyon field configuration will move also on these geodesic lines. We shall discuss this question in the following section. 


\section{Nonlinear electrodynamic world with oscillating bidyons}

In this section we shall discuss a possible world constructed from the great number of the bidyon-type field configurations. It is evident that in this world the bidyons and its possible strong coupling combinations may play the role of particles. Particles with full angular momentum multiples to $\frac{\hbar}{2}$ may be constructed from some number of the bidyons. It is amply clear that moving bidyon (9.34) has both particle and wave properties.

Because $\frac{\bar{d}}{\bar{b}}=\bar{\alpha} \ll 1$, we may build a perturbation theory with the fine structure constant $\bar{\alpha}$ as small parameter, for some aspects of the mathematical model of this world. Thus we have an analogy with perturbative procedure of quantum electrodynamics.

The world's field configuration is solution of the model's equations in whole fourdimensional space and each dyon singularity has a determined trajectory providing the satisfaction to the point boundary conditions. To obtain the solution for whole space, actually we may use the method of successive approximation only. According our method describing in section 0, the dyon's trajectories are corrected for each step of iteration with help of some integral conditions. In the present article we use the integral conservation law of the energy-momentum for this purpose. Thus this method for obtaining the trajectories is non-local in character. We have the classical generalized Lorentz force in initial approximation, for the case when we may consider the conservation law for the localization region of one dyon only. In general case the integral conditions may give the following effect. If we have a change of the field near some distant (from the trajectory) point, then we may obtain a change of this trajectory (without a propagation of disturbance from this distant point). This looks as quantum behavior when events at space separated points behave as though there is an instantaneous interaction between the events. According our concept, this pattern may be connected with the specific iterative way on which we go (in our calculations) to unknown world's field configuration in space-time.

The wave vector $k_{\mu}$ of moving quick-oscillating bidyon (9.34) (without any external given fields) satisfies the following dispersion relation:

$$
k^{\mu} k_{\mu}=h^{00} \underline{\omega}^{2} .
$$

We have also the following relation for the vector of full energy-momentum of the bidyon field configuration:

$$
P^{\mu} P_{\mu}=h^{00} m^{2},
$$

where $m$ is the full rest energy interpreted as mass.

However, because we have the non-restricted wave (with infinite three-dimensional energy integral) as a part of the bidyon configuration, how we must calculate the full 
energy-momentum in this case is not clear. A future consideration of the problem for motion of the bidyon with the associated quick-oscillating waves in given external field must give a value of the mass. We can denote the relation between the mass and the rest frequency as $\mathcal{C} \equiv m / \underline{\omega}$. Thus we have

$$
P_{\mu}=\mathcal{C} k_{\mu}
$$

To correlate between the bidyons (or multidyons) and real particles, we must have that $\mathcal{C}=\hbar$. To determine truth or falsity of this condition for the bidyon solution, we must have this solution and value of the mass. But the behavior of bidyon's initial approximation (9.1) at very small interdyonic distances (high energies) may suggest some optimism (see (9.27), (9.31)). Of course, outside of the regions immediately adjacent to the singular points the associated exact solution may be significantly different from the initial approximation. Thus this problem need further consideration.

Gravitational interaction (in the sense of bimetric gravitational theory) may exist for the bidyon solution. The quick-oscillating part of the solution with small amplitude will have tendency to move on geodesic line of induced by background field Riemann space. We may presume that in some cases this tendency will be a controlling factor for motion of the bidyon, that is the bidyon will be piloted by its small amplitude quick-oscillating part. This case may takes place when we have the quick-oscillating background field such that the classical electromagnetic interaction for the bidyon is absent because of averaging. We must presume also that the averaging cancels the effects of associated resonance interaction. But induced metric (10.1) includes squares of the field and the appropriate effect will not wipe out by averaging.

Thus we may hope that the nonlinear electrodynamic world will have a correlation with the real material world. In this case we could call an associated theory as Nonlinear Electrodynamic Theory of the World. Such theory may be considered as like Einstein's unified field theory with electromagnetic field as basic. In the context of this approach, all diversity of solutions and nonlinear effects for the electrodynamic field model must correlate with all real particles and their interactions. The evident practical significance of such approach consists in a possible discovery any new real effects with help of the theoretical investigation of the model. But whilst still we have more questions than answers on this arduous but feasible way.

\section{Conclusions}

Thus we have presented the initial theory for nonlinear Born-Infeld electrodynamics with dyonic singularities. We have proposed the method for investigation of interaction between the dyons. For a special case we have obtained the generalized Lorentz 
force acting on the dyon. We have introduced the concept of bidyon, interpreted as electromagnetic model of particle with spin, and we have investigated its initial approximation. We have obtained the effect of gravitational interaction, which appear within the limits of this electrodynamic model. We have also discussed possible correlations between the nonlinear electrodynamic world and real material world.

\section{References}

[1] A.A. Chernitskii, Long-range interaction of four-vector field solitons of the Minkowskian space, Theor. Math. Phys. 90(3) (1992) 260 (380 in Russian).

[2] A.A. Chernitskii, Gravitation as long-range interaction of solitons in non-linear electrodynamics, Book of abstracts GR14 (Florence) (1995), A96. .

[3] A.A. Chernitskii, Light beams distortion in nonlinear electrodynamics, J. High Energy Phys. 11 (1998) 015 hep-th/9809175.

[4] M. Born and L. Infeld, Foundations of the New Field Theory, Proc. Roy. Soc. 144 (1934) 425.

[5] A.S. Eddington, The Mathematical Theory of Relativity (Cambridge, 1924).

[6] A.A. Chernitskii, Nonlinear electrodynamics with singularities (Modernized BornInfeld electrodynamics), Helv. Phys. Acta 71 (1998) 274-287 hep-th/9705075.

[7] J. Schwinger, A Magnetic Model of Matter, Science 165 (1969) 757-761.

[8] I. Bialynicki-Birula, Nonlinear Electrodynamics: Variations on a Theme fy Born and Infeld, Quantum Theory of Particles and Fields ed.B. Jancewicz and J. Lukierski, (1983) 31-47.

[9] G. Gibbons, Electric-Magnetic Duality Rotations in Non-Linear Electrodynamics, Nucl. Phys. B454 (1995) 185-206 hep-th/9506035.

[10] P.A.M. Dirac, The theory of magnetic poles, Phys. Rev., Sec. ser. 74 (1948) 817-830.

[11] R. Curant and D. Hilbert, Method der Mathematical Physik, (Verlag von Julius Springer, Berlin, 1931).

[12] V.C.L. Huston and J.S. Pym, Applications of Functional Analysis and Operator Theory (Academic Press, London, 1980). 